\title{
CMC FOLIATIONS OF OPEN SPACETIMES ASYMPTOTIC TO OPEN ROBERTSON-WALKER SPACETIMES
}

\author{
CLAUS GERHARDT
}

\begin{abstract}
We consider open globally hyperbolic spacetimes $N$ of dimension $n+1, n \geq 3$, which are spatially asymptotic to a RobertsonWalker spacetime or an open Friedmann universe with spatial curvature $\tilde{\kappa}=0,-1$ and prove, under reasonable assumptions, that there exists a unique foliation by spacelike hypersurfaces of constant mean curvature and that the mean curvature function $\tau$ is a smooth time function if $N$ is smooth. Moreover, among the Friedmann universes which satisfy the necessary conditions are those that reflect the present assumptions of the development of the universe.
\end{abstract}

\section{Contents}

1. Introduction 1

2. Notations, assumptions and definitions 3

3. $C^{0}$-estimates 9

4. Gradient estimates 14

5. Existence of a solution of the Dirichlet problem 22

6. Decay estimates for the solutions 32

7. Existence of a CMC foliation 38

8. The constant mean curvature $\tau$ of the foliation by spacelike hypersurfaces is a smooth global time function 45

9. Appendix: Lipschitz continuous solutions are regular 58

$\begin{array}{ll}\text { References } & 60\end{array}$

\section{INTRODUCTION}

Foliating a Lorentzian manifolds $N=N^{n+1}$ by spacelike hypersurfaces of constant mean curvature (CMC hypersurfaces) and using the mean curvature $\tau$ of the foliation hypersurfaces as a time function is very important for physical models of the universe. Solving the Einstein equations is a lot easier if the initial hypersurface has constant mean curvature and in Friedmann

Date: August 27, 2020.

2000 Mathematics Subject Classification. 35J60, 53C21, 53C44, 53C50, 58J05.

Key words and phrases. Lorentzian manifold, open spacetimes, CMC foliation, general relativity. 
universes the mean curvature of the CMC hypersurfaces is also known as the Hubble constant - apart from a sign.

In case $N$ is globally hyperbolic and spatially compact, i.e., in case the Cauchy hypersurfaces are compact, the existence of a foliation by CMC hypersurfaces has been proved in [4]. If the Cauchy hypersurfaces are noncompact only trivial $\mathrm{CMC}$ foliations in Robertson-Walker spacetimes are known so far. In this paper we prove the existence of a $\mathrm{CMC}$ foliation in open globally hyperbolic spacetimes $N=N^{n+1}, n \geq 3$, which are spatially asymptotic to a Robertson-Walker spacetime $\hat{N}$. A Robertson-Walker spacetime is the warped product

$$
\hat{N}=I \times \hat{\mathcal{S}}_{0},
$$

where $\hat{\mathcal{S}}_{0}$ is a space of constant curvature and we consider the cases where $\hat{\mathcal{S}}_{0}$ is either $\mathbb{R}^{n}$ or $\mathbb{H}^{n}$. These assumptions on the Cauchy hypersurfaces are also favoured in present cosmological models where it is mostly assumed that $\hat{\mathcal{S}}_{0}=\mathbb{R}^{n}$.

Let $\hat{H}=\hat{H}(t)$ be the mean curvature of the slices

$$
\left\{x^{0}=t\right\}, \quad t \in I,
$$

in $\hat{N}$ with respect to the past directed normal, i.e.,

$$
\hat{H}=g^{i j} h_{i j}=-n \frac{a^{\prime}}{a},
$$

where $a$ is the scale factor and $h_{i j}$ the second fundamental form, then the only condition we impose on $\hat{N}$ is

$$
\hat{H}^{\prime}>0
$$

where a prime indicates differentiation with respect to $t$. This condition is satisfied by the models for an expanding Friedmann universe, see e.g., [8], where the expansion is driven by dark matter and dark energy densities.

The existence of a CMC foliation is achieved by first solving the Dirichlet problems

$$
\begin{aligned}
H_{\left.\right|_{M}} & =\hat{H}\left(t_{0}\right) \quad \text { in } B_{R}=B_{R}\left(\bar{x}_{0}\right), \\
u_{\left.\right|_{\partial B_{R}}} & =t_{0}
\end{aligned}
$$

for spacelike graphs, $M=\operatorname{graph} u$, over nested balls $B_{R} \subset \mathcal{S}_{0}$ with fixed center and expanding radii, and proving uniform a priori estimates in $C^{m, \alpha}\left(\bar{B}_{R}\right)$, $m \geq 3,0<\alpha<1$, independent of $R$ and then letting $R$ tend to infinity. We then obtain a unique foliation of $N$ by spacelike hypersurfaces

$$
M_{t}=\operatorname{graph} u((t, x))_{\left.\right|_{\mathcal{S}_{0}}}, \quad t \in I,
$$

having constant mean curvature $\hat{H}(t)$. The hypersurfaces $M_{t}$ uniformly converge to the slices

$$
\left\{x^{0}=t\right\}
$$


in $C^{m, \alpha}\left(\mathcal{S}_{0}\right)$ if $r(x)$ tends to infinity, where $r(x)$ is a radial distance function. Finally, the mean curvature $\tau$ of the foliation hypersurfaces is a smooth global time function if $N$ is smooth. Here, is a more formal statement of this result:

Theorem 1.1. The functions

$$
u(t, x), \quad(t, x) \in I \times \mathcal{S}_{0},
$$

describing the foliation by spacelike hypersurfaces $M_{t}, t \in I$, are of class $C^{m-3,1}$ in $t$ such that

$$
D_{t}^{k} u \in C^{m-k, \alpha}\left(\mathcal{S}_{0}\right) \quad \forall 0 \leq k \leq m-3,
$$

if $N$ is of class $C^{m, \alpha}, m \geq 3,0<\alpha<1$; if $N$ is smooth, i.e., $m=\infty$, then $u$ is also smooth in the variables $(t, x)$ and the mean curvature function $\tau=\tau\left(x^{0}, x\right)$ is a smooth time function.

\section{Notations, assumptions And DEFinitions}

The main objective of this section is to state the equations of Gauß, Codazzi, and Weingarten for spacelike hypersurfaces $M$ in a (n+1)-dimensional Lorentzian space $N$. Geometric quantities in $N$ will be denoted by $\left(\bar{g}_{\alpha \beta}\right),\left(\bar{R}_{\alpha \beta \gamma \delta}\right)$, etc., and those in $M$ by $\left(g_{i j}\right),\left(R_{i j k l}\right)$, etc. Greek indices range from 0 to $n$ and Latin from 1 to $n$; the summation convention is always used. Generic coordinate systems in $N$ resp. $M$ will be denoted by $\left(x^{\alpha}\right)$ resp. $\left(\xi^{i}\right)$. Covariant differentiation will simply be indicated by indices, only in case of possible ambiguity they will be preceded by a semicolon, i.e. for a function $u$ in $N,\left(u_{\alpha}\right)$ will be the gradient and $\left(u_{\alpha \beta}\right)$ the Hessian, but e.g., the covariant derivative of the curvature tensor will be abbreviated by $\bar{R}_{\alpha \beta \gamma \delta ; \epsilon}$. We also point out that

$$
\bar{R}_{\alpha \beta \gamma \delta ; i}=\bar{R}_{\alpha \beta \gamma \delta ; \epsilon} x_{i}^{\epsilon}
$$

with obvious generalizations to other quantities.

Let $M$ be a spacelike hypersurface, i.e. the induced metric is Riemannian, with a differentiable unit normal $\nu$ that is timelike.

In local coordinates, $\left(x^{\alpha}\right)$ and $\left(\xi^{i}\right)$, the geometric quantities of the spacelike hypersurface $M$ are connected through the following equations

$$
x_{i j}^{\alpha}=h_{i j} \nu^{\alpha}
$$

the so-called Gauß formula. Here, and also in the sequel, a covariant derivative is always a full tensor, i.e.,

$$
x_{i j}^{\alpha}=x_{, i j}^{\alpha}-\Gamma_{i j}^{k} x_{k}^{\alpha}+\bar{\Gamma}_{\beta \gamma}^{\alpha} x_{i}^{\beta} x_{j}^{\gamma} .
$$

The comma indicates ordinary partial derivatives.

In this implicit definition the second fundamental form $\left(h_{i j}\right)$ is taken with respect to $\nu$.

The second equation is the Weingarten equation

$$
\nu_{i}^{\alpha}=h_{i}^{k} x_{k}^{\alpha},
$$


where we remember that $\nu_{i}^{\alpha}$ is a full tensor.

Finally, we have the Codazzi equation

$$
h_{i j ; k}-h_{i k ; j}=\bar{R}_{\alpha \beta \gamma \delta} \nu^{\alpha} x_{i}^{\beta} x_{j}^{\gamma} x_{k}^{\delta}
$$

and the Gauß equation

$$
R_{i j k l}=-\left\{h_{i k} h_{j l}-h_{i l} h_{j k}\right\}+\bar{R}_{\alpha \beta \gamma \delta} x_{i}^{\alpha} x_{j}^{\beta} x_{k}^{\gamma} x_{l}^{\delta} .
$$

Now, let us assume that $N$ is a globally hyperbolic Lorentzian manifold with a compact Cauchy surface. $N$ is then a topological product $\mathbb{R} \times \mathcal{S}_{0}$, where $\mathcal{S}_{0}$ is a compact Riemannian manifold, and there exists a Gaussian coordinate system $\left(x^{\alpha}\right)$, such that the metric in $N$ has the form

$$
d \bar{s}_{N}^{2}=e^{2 \psi}\left\{-\left(d x^{0}\right)^{2}+\sigma_{i j}\left(x^{0}, x\right) d x^{i} d x^{j}\right\},
$$

where $\sigma_{i j}$ is a Riemannian metric, $\psi$ a function on $N$, and $x$ an abbreviation for the spacelike components $\left(x^{i}\right)$, cf. [3, Theorem 1.1]. We also assume that the coordinate system is future oriented, i.e. the time coordinate $x^{0}$ increases on future directed curves. Hence, the contravariant timelike vector $\left(\xi^{\alpha}\right)=(1,0, \ldots, 0)$ is future directed as is its covariant version $\left(\xi_{\alpha}\right)=e^{2 \psi}(-1,0, \ldots, 0)$.

Let $M=\operatorname{graph} u_{\left.\right|_{\mathcal{S}_{0}}}$ be the hypersurface

$$
M=\left\{\left(x^{0}, x\right): x^{0}=u(x), x \in \Omega \subset \mathcal{S}_{0}\right\},
$$

where $\Omega$ is an open domain in $\mathcal{S}_{0}$, then the induced metric has the form

$$
g_{i j}=e^{2 \psi}\left\{-u_{i} u_{j}+\sigma_{i j}\right\}
$$

where $\sigma_{i j}$ is evaluated at $(u, x)$, and its inverse $\left(g^{i j}\right)=\left(g_{i j}\right)^{-1}$ can be expressed as

$$
g^{i j}=e^{-2 \psi}\left\{\sigma^{i j}+\frac{u^{i}}{v} \frac{u^{j}}{v}\right\},
$$

where $\left(\sigma^{i j}\right)=\left(\sigma_{i j}\right)^{-1}$ and

$$
\begin{aligned}
u^{i} & =\sigma^{i j} u_{j} \\
v^{2} & =1-\sigma^{i j} u_{i} u_{j} \equiv 1-|D u|^{2} .
\end{aligned}
$$

Hence, graph $u$ is spacelike if and only if $|D u|<1$.

The covariant form of a normal vector of a graph looks like

$$
\left(\nu_{\alpha}\right)= \pm v^{-1} e^{\psi}\left(1,-u_{i}\right) .
$$

and the contravariant version is

$$
\left(\nu^{\alpha}\right)=\mp v^{-1} e^{-\psi}\left(1, u^{i}\right) .
$$

Thus, we have 
Remark 2.1. Let $M$ be spacelike graph in a future oriented coordinate system. Then, the contravariant future directed normal vector has the form

$$
\left(\nu^{\alpha}\right)=v^{-1} e^{-\psi}\left(1, u^{i}\right)
$$

and the past directed

$$
\left(\nu^{\alpha}\right)=-v^{-1} e^{-\psi}\left(1, u^{i}\right) .
$$

In the Gauß formula (2.2) we are free to choose the future or past directed normal, but we stipulate that we always use the past directed normal.

Look at the component $\alpha=0$ in (2.2) and obtain in view of (2.15)

$$
e^{-\psi} v^{-1} h_{i j}=-u_{i j}-\bar{\Gamma}_{00}^{0} u_{i} u_{j}-\bar{\Gamma}_{0 j}^{0} u_{i}-\bar{\Gamma}_{0 i}^{0} u_{j}-\bar{\Gamma}_{i j}^{0}
$$

Here, the covariant derivatives a taken with respect to the induced metric of $M$, and

$$
-\bar{\Gamma}_{i j}^{0}=e^{-\psi} \bar{h}_{i j}
$$

where $\left(\bar{h}_{i j}\right)$ is the second fundamental form of the hypersurfaces $\left\{x^{0}=\right.$ const $\}$.

An easy calculation shows

$$
\bar{h}_{i j} e^{-\psi}=-\frac{1}{2} \dot{\sigma}_{i j}-\dot{\psi} \sigma_{i j},
$$

where the dot indicates differentiation with respect to $x^{0}$.

Sometimes, we need a Riemannian reference metric, e.g. if we want to estimate tensors. Since the Lorentzian metric can be expressed as in (2.7), we define a Riemannian reference metric $\left(\tilde{g}_{\alpha \beta}\right)$ by

$$
\tilde{g}_{\alpha \beta} d x^{\alpha} d x^{\beta}=e^{2 \psi}\left\{\left(d x^{0}\right)^{2}+\sigma_{i j} d x^{i} d x^{j}\right\}
$$

and we abbreviate the corresponding norm of a vector field $\eta$ by

$$
\|\eta\|=\left(\tilde{g}_{\alpha \beta} \eta^{\alpha} \eta^{\beta}\right)^{1 / 2},
$$

with similar notations for higher order tensors.

Finally, let us recall the definition of an achronal set:

Definition 2.2. A subset $M \subset N$ is called achronal provided any timelike curve meets $M$ at most once. For details see [12, p. 413].

Let us now formulate the assumptions on $N$. $N$ is a globally hyperbolic spacetime of dimension $n+1$ which is spatially asymptotic to a RobertsonWalker spacetime $\hat{N}$, which is a warped product

$$
\hat{N}=I \times \hat{\mathcal{S}}_{0},
$$

where $\hat{\mathcal{S}}_{0}$ is either $\mathbb{R}^{n}$ or $\mathbb{H}^{n}$. The interval $I$ has the endpoints

$$
I=\left(t_{-}, t_{+}\right) .
$$

In physical applications $\hat{N}$ is a Friedmann universe. In [8] we proved the existence of an open Friedmann universe with

$$
I=(0, \infty)
$$


which has a big bang singularity and the mean curvature $\hat{H}(t)$ of the slices

$$
\left\{x^{0}=t\right\}
$$

is negative with respect to the past directed normal such that

$$
\hat{H}^{\prime} \geq c_{0}>0
$$

on compact subsets of $I$ and $\hat{H}$ satisfies

$$
\lim _{t \rightarrow 0} \hat{H}(t) \equiv H_{-}=-\infty
$$

as well as

$$
\lim _{t \rightarrow \infty} \hat{H}(t) \equiv H_{+}<0 .
$$

Since

$$
\hat{H}^{\prime}=|\hat{A}|^{2}+\hat{R}_{\alpha \beta} \nu^{\alpha} \nu^{\beta}=|\hat{A}|^{2}+\hat{R}_{00},
$$

where

$$
|\hat{A}|^{2}=\hat{h}^{i j} \hat{h}_{i j},
$$

we have

$$
|\hat{A}|^{2}+\hat{R}_{\alpha \beta} \nu^{\alpha} \nu^{\beta}>0
$$

while

$$
\hat{R}_{00}=-n \frac{\ddot{a}}{a}<0
$$

because

$$
\ddot{a}>0,
$$

where $a(t)$ is the scale factor.

Assumption 2.3 (Assumptions on $\hat{N}$ ). In this paper we do not assume that $\hat{N}$ is a Friedmann universe nor do we assume that $\hat{H}$ is negative but we require (2.25) on compact subsets of $I$ or equivalently (1.4). Then the limits

$$
\lim _{t \rightarrow t_{-}} \hat{H}(t) \equiv H_{-} \geq-\infty
$$

and

$$
\lim _{t \rightarrow t_{+}} \hat{H}(t) \equiv H_{+} \leq \infty
$$

exist, i.e., $\hat{N}$ has only to satisfy (2.25).

Since $N$ is supposed to be asymptotic to $\hat{N}$ we shall assume that both have the common time function $x^{0}$, that $N$ can also be written as a smooth product as in (2.21), though we shall write $\mathcal{S}_{0}$ instead of $\hat{\mathcal{S}}_{0}$ because we consider $\mathcal{S}_{0}$ to be an embedded Cauchy hypersurface which is diffeomorphic to $\hat{\mathcal{S}}_{0} . \quad N$ is supposed to be of class $C^{m, \alpha}, m \geq 3,0<\alpha<1$, i.e., the 
coordinate systems should be of class $C^{m, \alpha}$, the metric $\bar{g}_{\alpha \beta}$ of class $C^{m-1, \alpha}$ and the second fundamental form $\bar{h}_{i j}$ of the slices

$$
\left\{x^{0}=\text { const }\right\}
$$

of class $C^{m-2, \alpha}$. We assume that $N$ and $\hat{N}$ can be covered by a joint atlas of coordinate patches. The radial geodesic distance $\rho$ to a given point in $\hat{\mathcal{S}}_{0}$ is also defined in $\mathcal{S}_{0}$ but is of course only of class $C^{m, \alpha}$ there. In a coordinate slice

$$
\left\{x^{0}=t_{0}\right\}
$$

in $\hat{N}$ the geodesic distance would be

$$
r=a\left(t_{0}\right) \rho
$$

which is also defined when the corresponding slice is embedded in $N$.

The metric in $\hat{N}$ has the form

$$
d \hat{s}^{2}=-\left(d x^{0}\right)^{2}+\hat{\sigma}_{i j}\left(x, x^{0}\right) d x^{i} d x^{j},
$$

where

$$
\hat{\sigma}_{i j}=a^{2}\left(x^{0}\right) \tilde{\sigma}_{i j}(x)
$$

and $\tilde{\sigma}_{i j}$ is the metric in $\hat{\mathcal{S}}_{0}$.

The metric in $N$ can be written as

$$
\begin{aligned}
d \bar{s}^{2} & =e^{2 \psi}\left\{-\left(d x^{0}\right)^{2}+\sigma_{i j}\left(x, x^{0}\right) d x^{i} d x^{j}\right\} \\
& \equiv \bar{g}_{\alpha \beta} d x^{\alpha} d x^{\beta} .
\end{aligned}
$$

Let $\bar{h}_{i j}\left(x, x^{0}\right)$ resp. $\hat{h}_{i j}\left(x, x^{0}\right)$ be the second fundamental form of the slices

$$
\left\{x^{0}=\text { const }\right\}
$$

embedded in $N$ resp. $\hat{N}, e^{2 \psi} \sigma_{i j}$ resp. $\hat{\sigma}_{i j}$ the corresponding induced metrics and $\bar{\Gamma}_{i j}^{k}\left(x, x^{0}\right)$ resp. $\hat{\Gamma}_{i j}^{k}\left(x, x^{0}\right)$ the corresponding Christoffel symbols, then we shall assume:

Assumption 2.4 (Asymptotic behaviour). There exists $R_{0}>0$ and a constant $c>0$, which only depends on the compact sets in which $x^{0}$ ranges but not on $x$, provided

$$
r(x)>R_{0},
$$

or equivalently,

$$
\rho(x)>R_{0},
$$

such that

(2.44) $\sigma_{i j}\left(x, x^{0}\right)$ and $\hat{\sigma}_{i j}\left(x, x^{0}\right)$ are uniformly equivalent as long as $x^{0}$ ranges in a compact subset of $I$.

$$
\pm\left(\sigma_{i j}\left(x, x^{0}\right)-\hat{\sigma}_{i j}\left(x, x^{0}\right)\right) \leq \frac{c}{r(x)} \sigma_{i j}\left(x, x^{0}\right),
$$




$$
\begin{gathered}
\left|e^{\psi}-1\right| \leq \frac{c}{r(x)}, \\
\pm\left(\bar{h}_{i j}\left(x, x^{0}\right)-\hat{h}_{i j}\left(x, x^{0}\right)\right) \leq \frac{c}{r(x)} \sigma_{i j}\left(x, x^{0}\right), \\
\left|\bar{\Gamma}_{i j}^{k}\left(x, x^{0}\right)-\hat{\Gamma}_{i j}^{k}\left(x, x^{0}\right)\right| \leq \frac{c}{r(x)},
\end{gathered}
$$

where the norm on the left-hand side is an abbreviation for the norm of the corresponding tensor with respect to the metric $\sigma_{i j}\left(x, x^{0}\right)$. Furthermore, we assume

$$
\begin{gathered}
\left|D^{\gamma}\left(e^{\psi}-1\right)\right| \leq \frac{c}{r(x)} \quad \forall 0 \leq|\gamma| \leq m-1, \\
\mid D^{\gamma}\left(\bar{h}_{i j}\left(x, x^{0}\right)-\hat{h}_{i j}\left(x, x^{0}\right)\left|\leq \frac{c}{r(x)} \quad \forall 0 \leq\right| \gamma \mid \leq m-2,\right.
\end{gathered}
$$

and

$$
\left|D^{\gamma}\left(\bar{\Gamma}_{i j}^{k}-\hat{\Gamma}_{i j}^{k}\right)\right| \leq \frac{c}{r(x)} \quad \forall 0 \leq|\gamma| \leq m-2,
$$

where the derivatives are either partial derivatives with respect to $x^{0}$ or spatial covariant derivatives with respect to $\sigma_{i j}\left(x, x^{0}\right)$.

Remark 2.5. The previous assumptions on the asymptotic behaviour of $N$ and the assumption (2.25) imply

$$
\dot{\bar{H}}\left(x, x^{0}\right) \geq c_{0}>0 \quad \forall x \in\left\{r(x)>R_{0}\right\}
$$

uniformly in $x^{0}$ as long as $x^{0}$ stays in a compact subset of $I$.

Assumption 2.6 (Additional assumptions on $N$ ). $N$ should satisfy two additional assumptions. First, for any spacelike hypersurface of class $C^{2}$ we assume that

$$
|A|^{2}+\bar{R}_{\alpha \beta} \nu^{\alpha} \nu^{\beta} \geq 0 .
$$

Secondly, we assume the existence of future and past mean curvature barriers

$$
M_{k}=\operatorname{graph} \psi_{\left.k\right|_{\mathcal{S}_{0}}}, \quad k \in \mathbb{Z},
$$

with mean curvatures

$$
H_{k}=H_{\left.\right|_{M_{k}}},
$$

which are, for fixed $k$, uniformly spacelike hypersurfaces satisfying

$$
\begin{gathered}
\psi_{k} \in C^{3, \alpha}\left(\mathcal{S}_{0}\right), \quad \forall k \in \mathbb{Z}, \\
H_{-}<\inf _{\mathcal{S}_{0}} H_{\left.\right|_{M_{k}}} \leq \sup _{\mathcal{S}_{0}} H_{\left.\right|_{M_{k}}}<H_{+}, \quad \forall k \in \mathbb{Z}, \\
t_{-}=\lim _{k \rightarrow-\infty} \sup _{\mathcal{S}_{0}} \psi_{k}<\lim _{k \rightarrow \infty} \inf _{\mathcal{S}_{0}} \psi_{k}=t_{+},
\end{gathered}
$$


and

$$
H_{-}=\lim _{k \rightarrow-\infty} \sup _{M_{k}} H_{k}<\lim _{k \rightarrow \infty} \inf _{M_{k}} H_{k}=H_{+}
$$

where $t_{ \pm}$are the endpoints of $I$ and $H_{ \pm}$are the limits in Assumption 2.3. The previous assumptions on the $\psi_{k}$ also imply that we may assume without loss of generality

$$
k<l \Longrightarrow \sup _{\mathcal{S}_{0}} \psi_{k}<\inf _{\mathcal{S}_{0}} \psi_{l}
$$

and

$$
\sup _{M_{k}} H_{k}<\inf _{M_{l}} H_{l}
$$

otherwise we consider a subsequence.

\section{3. $C^{0}$-estimates}

In this section we want to derive a priori bounds for solutions of the Dirichlet problem

$$
\begin{aligned}
H_{\left.\right|_{M}} & =f(u, x) \quad \text { in } B_{R}\left(\bar{x}_{0}\right), \\
u_{\left.\right|_{\partial B_{R}}} & =t_{0}
\end{aligned}
$$

where

$$
M=\operatorname{graph} u=\left\{\left(x^{0}, x\right): x^{0}=u(x), \quad x \in B_{R}\left(\bar{x}_{0}\right)\right\}
$$

and

$$
B_{R} \equiv B_{R}\left(\bar{x}_{0}\right) \subset \mathcal{S}_{0} .
$$

$\mathcal{S}_{0}$ is a Cauchy hypersurface. We shall generally assume that $\mathcal{S}_{0}$ is a fixed coordinate slice

$$
\mathcal{S}_{0}=\left\{x^{0}=\bar{t}\right\}
$$

endowed with the induced metric

$$
e^{2 \psi(\bar{t}, \cdot)} \sigma_{i j}(\bar{t}, \cdot) .
$$

As a preparation let us first prove some lemmata.

Lemma 3.1. Let $M_{i}, i=1,2$, be spacelike hypersurfaces of class $C^{1}$ which are graphs over a bounded open domain $\Omega \subset \mathcal{S}_{0}$ such that

$$
\inf _{\partial \Omega} u_{2}>\sup _{\partial \Omega} u_{1}
$$

and suppose, furthermore, that there exists a broken future directed timelike curve $\gamma=\left(\gamma^{\alpha}\right)$ of class $C^{1}$ from $\bar{M}_{2}$ to $\bar{M}_{1}$, then the endpoints of $\gamma$ must both lie in the interior of the $M_{i}$. 
Proof. We argue by contradiction. Let the curve be parameterized over the interval $[0,1]$ such that

$$
\gamma(0)=p_{2}=\left(u\left(x_{2}\right), x_{2}\right) \in \bar{M}_{2} .
$$

and

$$
\gamma(1)=p_{1}=\left(u_{1}\left(x_{1}\right), x_{1}\right) \in \bar{M}_{1} .
$$

(i) First, assume that

$$
x_{2} \in \partial \Omega,
$$

then we have

$$
u_{1}\left(x_{1}\right)=\gamma^{0}(1)>\gamma^{0}(0)=u_{2}\left(x_{2}\right)
$$

and hence

$$
\gamma(1) \in M_{1},
$$

in view of the assumption (3.6). Let $\tau_{0}$ be the largest $\tau$ such that

$$
\left(\gamma^{i}(\tau)\right) \in \partial \Omega,
$$

then

$$
0 \leq \tau_{0}<1
$$

and

$$
\gamma^{0}\left(\tau_{0}\right)>\sup _{\partial \Omega} u_{1},
$$

because of (3.6), from which we infer that there exists a future directed timelike curve $\tilde{\gamma}$ parameterized over the interval $[0,1]$ such that

$$
\tilde{\gamma}(0) \in M_{1}
$$

and

$$
\tilde{\gamma}(1)=\gamma(1) \in M_{1},
$$

such that $\tilde{\gamma}$ is completely contained in the open cylinder

$$
Q=I \times \Omega
$$

which will lead to a contradiction as we shall show in the lemma below.

$\tilde{\gamma}$ can be defined as follows: $\tilde{\gamma}(0)$ is connected to $\gamma(\tau)$ for some $\tau>\tau_{0}$ by the timelike curve

$$
\tilde{\gamma}(s)=\left(s \gamma^{0}(\tau)+(1-s) u_{1}\left(x_{0}\right), x_{0}\right),
$$

where

$$
x_{0}=\left(\gamma^{i}(\tau)\right) \in \Omega, \quad \tau>\tau_{0},
$$

and $\tau$ has to satisfy

$$
\gamma^{0}(\tau)>u_{1}\left(\gamma^{i}(\tau)\right)
$$

which is valid if

$$
0<\tau-\tau_{0}<\delta
$$


and $\delta$ small enough.

After this first segment $\tilde{\gamma}$ is identical with $\gamma$. A reparameterization of the first segment by setting

$$
\tilde{s}=\tau s
$$

then leads the final definition of $\tilde{\gamma}$.

(ii) Next, let us suppose

$$
x_{1} \in \partial \Omega,
$$

then

$$
\gamma(0) \in M_{2} .
$$

Let

$$
\tau_{0} \in(0,1]
$$

be the first $\tau$ such satisfying

$$
\left(\gamma^{i}(\tau)\right) \in \partial \Omega
$$

then

$$
\gamma^{0}\left(\tau_{0}\right) \leq \gamma^{0}\left(\tau_{1}\right)<\inf _{\partial \Omega} u_{2}
$$

and we conclude that there exists a future directed timelike curve $\tilde{\gamma}$ from

$$
\gamma(0)=p_{2} \in M_{2}
$$

to a point

$$
\tilde{\gamma}(1) \in M_{2}
$$

which lies completely inside the cylinder $Q$; again a contradiction.

$\tilde{\gamma}$ is similarly defined as before, only, that now its last segment has to be defined by

$$
\tilde{\gamma}(s)=\left(s u_{2}\left(\gamma^{i}(\tau)\right)+(1-s) \gamma^{0}(\tau), \gamma^{i}(\tau)\right)
$$

for some $\tau<\tau_{0}$, where $\tau$ has to satisfy

$$
u_{2}\left(\gamma^{i}(\tau)\right)>\gamma,
$$

which is valid if

$$
0<\tau_{0}-\tau<\delta
$$

and $\delta$ is small enough.

Lemma 3.2. Let $M$ be a spacelike graph over an open bounded domain $\Omega \subset \mathcal{S}_{0}$,

$$
M=\operatorname{graph} u,
$$

and assume that

$$
u \in C^{1}(\bar{\Omega}) .
$$


Then, $M$ is achronal in the open cylinder

$$
Q=I \times \Omega .
$$

Proof. For the definition of achronal confer Definition 2.2 on page 5. We argue by contradiction. Let $\gamma=\left(\gamma^{\alpha}\right)$ be a possibly broken timelike $C^{1}$-curve with image

$$
\Gamma=\{\gamma(\tau): 0 \leq \tau \leq 1\}
$$

and endpoints in $M$. Moreover, suppose that

$$
\Gamma \subset Q \text {. }
$$

Without loss of generality let us assume that $\gamma$ is future directed. Since $M$ is a $C^{1}$-graph it has a continuous timelike normal vector $\nu$ which we assume to be future directed. The open set

$$
U^{+}=\left\{\left(x^{0}, x\right): x^{0}>u(x), \quad x \in \Omega\right\}
$$

lies in the future of $M$ and

$$
U^{-}=\left\{\left(x^{0}, x\right): x^{0}<u(x), \quad x \in \Omega\right\}
$$

in the past of $M$.

Let $\tau_{0}$ be the first $\tau>0$ such that

$$
\gamma(\tau) \in M
$$

then the curve

$$
\Gamma_{0}=\left\{\gamma(\tau): 0 \leq \tau \leq \tau_{0}\right\}
$$

intersects $M$ exactly twice, namely, at its endpoints. Since $\gamma$ is future directed we deduce that there exists $\delta>0$ such that

$$
\gamma(\tau) \in U^{+} \quad \forall 0<\tau<\delta
$$

and

$$
\gamma(\tau) \in U^{-} \quad \forall \tau_{0}-\delta<\tau<\delta
$$

and we conclude that

$$
\gamma^{0}(\tau)-u\left(\gamma^{i}(\tau)\right)>0 \quad \forall 0<\tau<\delta
$$

and

$$
\gamma^{0}(\tau)-u\left(\gamma^{i}(\tau)\right)<0 \quad \forall \tau_{0}-\delta<\tau<\tau_{0},
$$

hence, there exists

$$
\tau_{1} \in\left(0, \tau_{0}\right)
$$

such that

$$
\gamma^{0}\left(\tau_{1}\right)=u\left(\gamma^{i}\left(\tau_{1}\right)\right)
$$

contradicting the fact that only the endpoints are part of $M$.

We are now ready to prove the crucial comparison theorem: 
Theorem 3.3. Let $\Omega \subset \mathcal{S}_{0}$ be a bounded open domain and let

$$
M_{i}=\operatorname{graph} u_{i}, \quad i=1,2,
$$

be spacelike graphs over $\Omega$ satisfying

$$
\begin{gathered}
u_{i} \in C^{2}(\bar{\Omega}), \\
\inf _{\Omega} H_{\left.\right|_{M_{2}}}>\sup _{\Omega} H_{\left.\right|_{M_{1}}},
\end{gathered}
$$

and

$$
\inf _{\partial \Omega} u_{2}>\sup _{\partial \Omega} u_{1}
$$

then

$$
u_{1}(x)<u_{2}(x) \quad \forall x \in \Omega .
$$

Proof. First, we observe that the weaker conclusion

$$
u_{1} \leq u_{2}
$$

is as good as the stricter inequality (3.52) because of the weak Harnack inequality. Hence, suppose that (3.53) is not valid so that

$$
E=\left\{x \in \bar{\Omega}: u_{2}(x)<u_{1}(x)\right\} \neq \emptyset .
$$

Then, there exist points $p_{i} \in \bar{M}_{i}$ such that

$$
\begin{aligned}
0<d_{0} & =d\left(\bar{M}_{2}, \bar{M}_{1}\right)=d\left(p_{2}, p_{1}\right) \\
& =\sup \left\{d(p, q):(p, q) \in \bar{M}_{2} \times \bar{M}_{1}\right\},
\end{aligned}
$$

where $d$ is the Lorentzian distance function which is continuous in globally hyperbolic spacetimes, cf. [2, Lemma 4.5, p. 140]. Let $\gamma=\left(\gamma^{\alpha}\right)$ be a maximal future directed geodesic from $\bar{M}_{2}$ to $\bar{M}_{1}$ realizing the distance with endpoints $p_{i} \in \bar{M}_{i}, i=1,2$, parameterized by arc length. Then, we first observe

$$
p_{i} \in M_{i}, \quad i=1,2,
$$

in view of Lemma 3.1.

We are now able to argue as in the proof of a corresponding result in $[7$, Lemma 4.7.1] to conclude that

$$
H_{\left.\right|_{M_{1}}}\left(p_{1}\right) \geq H_{\left.\right|_{M_{2}}}\left(p_{2}\right),
$$

because of the assumption (2.53) on page 8, contradicting (3.50).

As a corollary we obtain:

Corollary 3.4. Let $\Omega \subset \mathcal{S}_{0}$ be a bounded open domain and $M=\operatorname{graph} u$ be a spacelike hypersurface, where $u \in C^{2}(\bar{\Omega})$ is a solution of the Dirichlet problem in $\Omega$

$$
\begin{aligned}
& H_{\left.\right|_{M}}=f(u, x), \\
& u_{\left.\right|_{\partial \Omega}}=\varphi,
\end{aligned}
$$


satisfying

$$
H_{-}<\inf _{\Omega} f(u, x) \leq \sup _{\Omega} f(u, x)<H_{+}
$$

and

$$
t_{-}<\inf _{\partial \Omega} \varphi \leq \sup _{\partial \Omega} \varphi<t_{+}
$$

then $u$ is a priori bounded if the assumption (2.53) on page 8 is satisfied.

Proof. The proof follows immediately by employing appropriate barriers.

\section{Gradient estimates}

Let

$$
M=\operatorname{graph} u
$$

be a solution of the Dirichlet problem (3.1) on page 9 of class $C^{3}\left(\bar{B}_{R}\right)$. The right-hand side $f=f\left(x^{0}, x\right)$ should be of class $C^{1}$

$$
f \in C^{1}\left(I \times \bar{B}_{R}\right),
$$

though the gradient estimates will not depend on $\frac{\partial f}{\partial x^{0}}$ if

$$
\frac{\partial f}{\partial x^{0}} \leq 0
$$

which is important when the penalization method is applied to approximate solutions of variational inequalities. We shall employ this method in the next section when the existence of solutions to the Dirichlet problem is established. The fact that we consider a geodesic ball is of no importance. We could have chosen any precompact domain

$$
\Omega \subset \mathcal{S}_{0}
$$

with $\partial \Omega$ of class $C^{2}$. However, it is important that we consider constant boundary values for otherwise gradient estimates up to the boundary would be more difficult and the method we employ would fail. In [1, Prop. 3.2] it is proved that non-constant boundary values can be reduced to the constant case if certain conditions are satisfied but these conditions cannot be verified easily.

We are going to prove the following theorem:

Theorem 4.1. Let $M=$ graph $u$ be a solution of the Dirichlet problem (3.1) and let $K \subset N$ be a compact set such that $M \subset K$, then

$$
\tilde{v}=v^{-1}=\left(1-|D u|^{2}\right)^{-1}
$$

is uniformly bounded in $\bar{B}_{R}$,

$$
\tilde{v} \leq c
$$


where $c$ depends on $K, t_{0}$ and the values $|f|,|||D f|||$ and ambient curvature terms in $K$ as well as the supremum norm of the mean curvature $\hat{H}_{R}$ of $\partial B_{R}$. If $K$ is bounded by coordinate slices

$$
t_{1} \leq x^{0} \leq t_{2} \quad \forall\left(x^{0}, x\right) \in K
$$

then $c$ depends on

$$
c=c\left(t_{1}, t_{2},|f|_{\mathscr{S}},\|D f\|_{\mathscr{S}},\left|\hat{H}_{R}\right|_{\mathscr{S}}\right)
$$

and ambient curvature terms in

$$
\mathscr{S}=\mathscr{S}\left(t_{1}, t_{2}\right)
$$

where $\mathscr{S}$ is the region defined by

$$
\mathscr{S}=\left\{t_{1} \leq x^{0} \leq t_{2}\right\}
$$

provided the indicated norms in $\mathscr{S}$ are bounded. Since this is true in our case we can state that the a priori estimate is independent of $R$.

The proof of the theorem requires some preparatory steps.

Lemma 4.2. Let $M=$ graph $u$ satisfy the equation (3.1) on page 9, then $\tilde{v}$ satisfies the elliptic differential equation

$$
\begin{aligned}
-\Delta \tilde{v}= & -\|A\|^{2} \tilde{v}-f \eta_{\alpha \beta} \nu^{\alpha} \nu^{\beta} \\
& -2 h^{i j} x_{i}^{\alpha} x_{j}^{\beta} \eta_{\alpha \beta}-g^{i j} \eta_{\alpha \beta \gamma} x_{i}^{\beta} x_{j}^{\gamma} \nu^{\alpha} \\
& -\bar{R}_{\alpha \beta} \nu^{\alpha} x_{k}^{\beta} \eta_{\gamma} x_{l}^{\gamma} g^{k l}-f_{\beta} x_{i}^{\beta} \eta_{\alpha} x_{k}^{\alpha} g^{i k},
\end{aligned}
$$

where $\eta$ is the covariant vector field $\left(\eta_{\alpha}\right)=e^{\psi}(-1,0, \ldots, 0)$ and the covariant derivatives are to be understood with respect to the induced metric $g_{i j}$ on $M$.

Proof. We have $\tilde{v}=\langle\eta, \nu\rangle$. Let $\left(\xi^{i}\right)$ be local coordinates for $M$. Differentiating $\tilde{v}$ covariantly we deduce

$$
\begin{gathered}
\tilde{v}_{i}=\eta_{\alpha \beta} x_{i}^{\beta} \nu^{\alpha}+\eta_{\alpha} \nu_{i}^{\alpha}, \\
\tilde{v}_{i j}=\eta_{\alpha \beta \gamma} x_{i}^{\beta} x_{j}^{\gamma} \nu^{\alpha}+\eta_{\alpha \beta} x_{i j}^{\beta} \nu^{\alpha} \\
+\eta_{\alpha \beta} x_{i}^{\beta} \nu_{j}^{\alpha}+\eta_{\alpha \beta} x_{j}^{\beta} \nu_{i}^{\alpha}+\eta_{\alpha} \nu_{i j}^{\alpha}
\end{gathered}
$$

Using then the Gauß formula, the Weingarten equation and the Ricci identities we obtain the desired result.

Lemma 4.3. Let $K \subset N$ be compact und $M \subset K$, then there is a constant $c=c(K)$ such that for any positive function $0<\epsilon=\epsilon(x)$ on $B_{R}$ we have

$$
\begin{aligned}
\|\nu\| & \leq c \tilde{v}, \\
g^{i j} & \leq c \tilde{v}^{2} \sigma^{i j},
\end{aligned}
$$


and

$$
\left|h^{i j} \eta_{\alpha \beta} x_{i}^{\alpha} x_{j}^{\beta}\right| \leq \frac{\epsilon}{2}\|A\|^{2} \tilde{v}+\frac{c}{2 \epsilon} \tilde{v}^{3}
$$

where $\left(\eta_{\alpha}\right)$ is the vector field in Lemma 4.2 and where we employed the Riemannian reference metric $\tilde{g}$.

Proof. The first two estimates can be immediately verified. To prove (4.16) we choose local coordinates $\left(\xi^{i}\right)$ such that

$$
h_{i j}=\kappa_{i} \delta_{i j}, \quad g_{i j}=\delta_{i j}
$$

and deduce

$$
\begin{aligned}
\left|h^{i j} \eta_{\alpha \beta} x_{i}^{\alpha} x_{j}^{\beta}\right| & \leq \sum_{i}\left|\kappa_{i}\right|\left|\eta_{\alpha \beta} x_{i}^{\alpha} x_{i}^{\beta}\right| \\
& \leq \frac{\epsilon}{2}\|A\|^{2} \tilde{v}+\frac{1}{2 \epsilon} \tilde{v}^{-1} \sum_{i}\left|\eta_{\alpha \beta} x_{i}^{\alpha} x_{i}^{\beta}\right|^{2},
\end{aligned}
$$

and

$$
\sum_{i}\left|\eta_{\alpha \beta} x_{i}^{\alpha} x_{i}^{\beta}\right|^{2} \leq g^{i k} \eta_{\alpha \beta} x_{i}^{\alpha} x_{j}^{\beta} g^{j l} \eta_{\gamma \delta} x_{k}^{\gamma} x_{l}^{\delta} .
$$

Hence, the result in view of (4.15).

Combining the preceding lemmata we infer

Lemma 4.4. There is a constant $c=c(K)$ such that for any positive function $\epsilon=\epsilon(x)$ on $B_{R}$ the term $\tilde{v}$ satisfies a parabolic inequality of the form

$$
-\Delta \tilde{v} \leq-(1-\epsilon)\|A\|^{2} \tilde{v}+c[|f|+\|D f \mid\|] \tilde{v}^{2}+c\left[1+\epsilon^{-1}\right] \tilde{v}^{3} .
$$

We note that the statement $c$ depends on $K$ also implies that $c$ depends on geometric quantities of the ambient space restricted to $K$.

We further need the following two lemmata

Lemma 4.5. Let $M=$ graph $u$ have prescribed mean curvature $f$, then

$$
-\Delta u=e^{-\psi} v^{-1} f-e^{-\psi} g^{i j} \bar{h}_{i j}+\bar{\Gamma}_{00}^{0}\|D u\|^{2}+2 \bar{\Gamma}_{0 i}^{0} u^{i} .
$$

Proof. This follows immediately from equation (2.16).

Lemma 4.6. Let $M \subset K$ be a graph over $B_{R}, M=$ graph $u$, then

$$
\left|\tilde{v}_{i} u^{i}\right| \leq c \tilde{v}^{3}+\|A\| e^{\psi}\|D u\|^{2},
$$

where $c=c(K)$. 
Proof. First, we use that

$$
\tilde{v}^{2}=1+e^{2 \psi}\|D u\|^{2}
$$

and thus,

$$
2 \tilde{v} \tilde{v}_{i}=2 \psi_{\alpha} x_{i}^{\alpha} e^{2 \psi}\|D u\|^{2}+2 e^{2 \psi} u_{i j} u^{j},
$$

from which we infer

$$
\left|\tilde{v}_{i} u^{i}\right| \leq c \tilde{v}^{3}+\tilde{v}^{-1} e^{2 \psi}\left|u_{i j} u^{i} u^{j}\right|,
$$

which gives the result because of (2.16).

We are now ready to prove Theorem 4.1.

Proof of Theorem 4.1. Let $\mu, \lambda$ be positive constants, where $\mu$ is supposed to be small and $\lambda$ large, and define

$$
\varphi=e^{\mu e^{\lambda(u+c)}}
$$

where $c$ is large positive constant such that $u+c>1$.

We shall show that

$$
w=\tilde{v} \varphi
$$

is uniformly bounded if $\mu, \lambda$ are chosen appropriately. Let $p_{0}=\left(u\left(y_{0}\right), y_{0}\right) \in$ $\bar{M}$ be point where $w$ attains its supremum

$$
w\left(p_{0}\right)=\sup _{\bar{M}} w,
$$

where we now consider the functions to be defined on $M$. We shall apply the maximum principle, or, at the boundary, a slight modification of it, to obtain an a priori bound for $w$.

(i) Let us first assume that $p_{0} \in M$, then we can apply the maximum principle directly. In view of Lemma 4.3 and Lemma 4.5 we have

$$
-\Delta \varphi \leq c \mu \lambda e^{\lambda(u+c)}\left[\tilde{v}|f|+\tilde{v}^{2}\right] \varphi-\mu \lambda^{2} e^{\lambda(u+c)}\left[1+\mu e^{\lambda(u+c)}\right]\|D u\|^{2} \varphi,
$$

from which we further deduce taking Lemma 4.4 and Lemma 4.6 into account

$$
\begin{aligned}
-\Delta w \leq & -(1-\epsilon)\|A\|^{2} \tilde{v} \varphi+c[|f|+\|D f\| \|] \tilde{v}^{2} \varphi \\
& +c\left[1+\epsilon^{-1}\right] \tilde{v}^{3} \varphi-\mu \lambda^{2} e^{\lambda(u+c)}\left[1+\mu e^{\lambda(u+c)}\right] \tilde{v}\|D u\|^{2} \varphi \\
& +c[1+|f|] \mu \lambda e^{\lambda(u+c)} \tilde{v}^{3} \varphi+2 \mu \lambda e^{\lambda(u+c)}\|A\| e^{\psi}\|D u\|^{2} \varphi .
\end{aligned}
$$

We estimate the last term on the right-hand side by

$$
\begin{aligned}
2 \mu \lambda e^{\lambda(u+c)}\|A\| e^{\psi}\|D u\|^{2} \varphi \leq & (1-\epsilon)\|A\|^{2} \tilde{v} \varphi \\
& +\frac{1}{1-\epsilon} \mu^{2} \lambda^{2} e^{2 \lambda(u+c)} \tilde{v}^{-1} e^{2 \psi}\|D u\|^{4} \varphi
\end{aligned}
$$


and conclude

$$
\begin{aligned}
-\Delta w \leq & c\left[|f|+|\|D f \mid\|] \tilde{v}^{2} \varphi+c[1+|f|] \mu \lambda e^{\lambda(u+c)} \tilde{v}^{3} \varphi\right. \\
& +c\left[1+\epsilon^{-1}\right] \tilde{v}^{3} \varphi+\left[\frac{1}{1-\epsilon}-1\right] \mu^{2} \lambda^{2} e^{2 \lambda(u+c)}\|D u\|^{2} \tilde{v} \varphi \\
& -\mu \lambda^{2} e^{\lambda(u+c)}\|D u\|^{2} \tilde{v} \varphi,
\end{aligned}
$$

where we have used that

$$
e^{2 \psi}\|D u\|^{2} \leq \tilde{v}^{2}
$$

Setting $\epsilon=e^{-\lambda(u+c)}$, we then obtain

$$
\begin{aligned}
-\Delta w \leq & c\left[|f|+|\|D f \mid\|] \tilde{v}^{2} \varphi+c e^{\lambda(u+c)} \tilde{v}^{3} \varphi\right. \\
& +c[1+|f|] \mu \lambda e^{\lambda(u+c)} \tilde{v}^{3} \varphi \\
& +\left[\frac{\mu}{1-\epsilon}-1\right] \mu \lambda^{2} e^{\lambda(u+c)}\|D u\|^{2} \tilde{v} \varphi .
\end{aligned}
$$

Now, we choose $\mu=\frac{1}{2}$ and $\lambda_{0}$ so large that

$$
\frac{\mu}{1-e^{-\lambda(u+c)}} \leq \frac{3}{4} \quad \forall \lambda \geq \lambda_{0}
$$

and infer that the last term on the right-hand side of (4.34) is less than

$$
-\frac{1}{8} \lambda^{2} e^{\lambda(u+c)}\|D u\|^{2} \tilde{v} \varphi
$$

which in turn can be estimated from above by

$$
-c \lambda^{2} e^{\lambda(u+c)} \tilde{v}^{3} \varphi
$$

at points where $\tilde{v} \geq 2$.

Thus, we conclude that for

$$
\lambda \geq \max \left(\lambda_{0}, 4\left[1+|f|_{K}\right]\right)
$$

the maximum principle, applied to $w$ at $y_{0}$, yields

$$
w \leq \operatorname{const}\left(\lambda_{0},|f|,\|D f\|, K\right) .
$$

Remark 4.7. The estimate (4.39) is also valid if $\varphi$ is replaced by

$$
\tilde{\varphi}=e^{\mu e^{-\lambda\left(u-c+2 t_{0}\right)}}
$$

and then considering

$$
\tilde{w}=\tilde{v} \tilde{\varphi}
$$

instead of $w$, where $\lambda, \mu$ and $c$ are defined as before. If $\tilde{w}$ attains its supremum at an interior point $p_{0} \in M$, then the inequality (4.39) is also valid if $w$ is replaced by $\tilde{w}$ on the left-hand side. 
(ii) We now assume that the supremum of $w$ is attained on the boundary of $M$, where

$$
u=t_{0},
$$

i.e., $u$ is constant on $\partial M$. We then argue similarly as Bartnik in the proof of [1, Theorem 3.1]. Consider $w$ and $\tilde{w}$ simultaneously. If one of the functions attains its maximum in the interior, then the estimate is already proved. Thus, let us assume that both attain their maximum on $\partial M$. Since

$$
w=\tilde{w} \quad \text { on } \partial M
$$

we consider a point $p_{0} \in \partial M$, or equivalently, $y_{0} \in \partial B_{R}$, such that $w$ and $\tilde{w}$ both attain their maximum in $p_{0}$. We may also assume that

$$
D u\left(y_{0}\right) \neq 0,
$$

for otherwise we have nothing to prove. Hence, either

or

$$
\frac{D u}{\|D u\|}
$$

$$
-\frac{D u}{\|D u\|}
$$

is equal to the outer normal $\tilde{\nu}$ of $\partial M$, i.e.,

$$
\tilde{\nu} \in T_{p_{0}}(M) \text {, }
$$

since $u$ is constant on $\partial M$. Let us assume that

$$
\tilde{\nu}=-\frac{D u}{\|D u\|}
$$

then we have in $p_{0}$

$$
0 \leq w_{i} \tilde{\nu}^{i}=\tilde{v}_{i} \tilde{\nu}^{i} \varphi+\tilde{v} \lambda e^{\lambda(u+c)} \varphi u_{i} \tilde{\nu}^{i}
$$

from which we deduce

$$
\begin{aligned}
\lambda e^{\lambda(u+c)} \tilde{v}\|D u\| & \leq \varphi\left|\tilde{v}_{i} u^{i}\right|\|D u\|^{-1} \\
& =\varphi\left|\tilde{v}_{i} \tilde{u}^{i}\right| \tilde{v}^{2} e^{-2 \psi}\|D u\|^{-1},
\end{aligned}
$$

where $\check{u}^{i}$ indicates that the index is raised with respect to the metric $\sigma_{i j}(u, x)$

$$
\check{u}^{i}=\sigma^{i j} u_{j}
$$

and where we recall that

$$
u^{i}=\tilde{v}^{2} e^{-2 \psi} \check{u}^{i},
$$

cf. [7, equ. (5.4.20) \& (5.4.21)].

On the other hand,

$$
\left|\tilde{v}_{i} \breve{u}^{i}\right| \leq c|f|+c|\bar{A}| \tilde{v}+c|| D \psi||+c|\mathscr{H}| \tilde{v}
$$

where the constant $c$ only depends on geometric quantities of the ambient metric in the capped region $\mathscr{S}\left(t_{1}, t_{2}\right)$ and where $\mathscr{H}$ is the mean curvature of the geodesic sphere $\partial B_{R}$ embedded in $\left(\mathcal{S}_{0}, \sigma_{i j}\left(t_{0}, x\right)\right), \bar{A}$ is the second 
fundamental form of the coordinate slice $M_{t_{0}}$. A proof is given in the lemma below.

Since $|\mathscr{H}|,|\bar{A}|,|f|$ and $|\psi|,|| D \psi \mid \|$ are uniformly bounded in $\mathscr{S}$ we conclude

$$
\left|\tilde{v}_{i} \check{u}^{i}\right| \leq c_{0} \tilde{v}
$$

if $\tilde{v}>2$ and hence we deduce

$$
\tilde{v}\left(p_{0}\right)<2
$$

if $\lambda$ is larger than some constant $\lambda_{0}$, where

$$
\lambda_{0}=\lambda_{0}(|\mathscr{H}|,|\bar{A}|,|f|,|\psi|,|| D \psi|\|,| u \mid)
$$

and where the norms on the right-hand side are supremum norms over $\partial B_{R}$ resp. $\mathscr{S}$ or $B_{R}$. Hence, we finally proved the a priori estimate for (4.6) in view of the result in the lemma below.

Lemma 4.8. Assume that $w$ attains its maximum at a point $p_{0}=$ $\left(u\left(y_{0}\right), y_{0}\right)$ with $y_{0} \in \partial B_{R}$ and that

$$
-\frac{\check{u}^{i}}{|D u|}=\hat{\nu}^{i},
$$

where $\hat{\nu}^{i}$ is the outward normal to $\partial B_{R}$. Suppose, furthermore, that $\tilde{v} \geq 2$, then the estimate (4.54) is valid.

Proof. First, let $\tilde{g}_{\alpha \beta}$ be the conformal metric to $\bar{g}_{\alpha \beta}$ such that

$$
\bar{g}_{\alpha \beta}=e^{2 \psi} \tilde{g}_{\alpha \beta}
$$

and consider $M$ to be embedded in $N$ equipped with the metric $\tilde{g}_{\alpha \beta}$ instead of $\bar{g}_{\alpha \beta}$. Denote the corresponding geometric quantities of $M$ by $\tilde{g}_{i j}, \tilde{h}_{i j}, \tilde{H}$ and $\tilde{\nu}=\left(\tilde{\nu}^{\alpha}\right)$. Then, we have

$$
e^{\psi} H=\tilde{H}+n \psi_{\alpha} \tilde{\nu}^{\alpha},
$$

cf. [7, equ. (1.1.52) on p. 7], and

$$
g^{i j}=e^{-2 \psi} \tilde{g} i j=e^{-2 \psi}\left(\sigma^{i j}+\tilde{v}^{2} \check{u}^{i} \check{u}^{j}\right) .
$$

Let $u_{i j}$ be the second covariant derivatives of $u$ with respect to the metric $\tilde{g}_{i j}$ and $u_{; i j}$ the covariant derivatives with respect to the metric $\sigma_{i j}(x, u)$, then

$$
u_{i j}=\tilde{v}^{2} u_{; i j},
$$

cf. [7, Lemma 2.7.6].

Next, let us differentiate

$$
\tilde{v}=\left(1-|D u|^{2}\right)^{-1}
$$

covariantly with respect to the metric $\sigma_{i j}(x, u)$ yielding

$$
\tilde{v}_{i}=\tilde{v}^{3} u_{; i j} \check{u}^{j}
$$

and hence

$$
\tilde{v}_{i} \check{u}^{i}=\tilde{v}^{3} u_{; i j} \check{u}^{i} \check{u}^{j}=\tilde{v}^{3} u_{; i j} \hat{\nu}^{i} \hat{\nu}^{j}|D u|^{2} .
$$


From (4.61) we infer that

$$
\begin{aligned}
\tilde{v} H & =-\tilde{v}^{2} \tilde{g}^{i j} u_{; i j}+\tilde{g}^{i j} \bar{h}_{i j} \\
& =-\tilde{v}^{2}\left\{\sigma^{i j} u_{; i j}+u_{; i j} \check{u}^{i} \check{u}^{j} \tilde{v}^{2}\right\}+\tilde{g}^{i j} \bar{h}_{i j},
\end{aligned}
$$

where $\bar{h}_{i j}$ is the second fundamental form of the coordinate slices with respect to the ambient metric $\tilde{g}_{\alpha \beta}$. Choosing a coordinate system $\left(x^{i}\right)$ in $y_{0} \in \partial B_{R}$ such that

$$
\begin{aligned}
\sigma_{i j} & =\delta_{i j}, \\
\frac{\partial}{\partial x^{n}} & =\hat{\nu},
\end{aligned}
$$

and $T_{y_{0}}\left(\partial B_{R}\right)$ is spanned by

$$
\frac{\partial}{\partial x^{i}}, \quad 1 \leq i \leq n-1,
$$

we see that we only have to estimate the tangential second derivatives of $u$ appropriately to complete the proof of the lemma.

Let $x^{i}\left(\xi^{a}\right), 1 \leq a \leq n-1$, be a local embedding of $\partial B_{R}$ into $\left(\mathcal{S}_{0}, \sigma_{i j}\left(t_{0}, x\right)\right)$ around $y_{0}=x\left(\xi_{0}\right)$, then

$$
0=u_{a}=u_{i} x_{a}^{i}
$$

and

$$
0=u_{a b}=u_{: i j} x_{a}^{i} x_{b}^{j}-\hat{h}_{a b} \hat{u}_{i} \nu^{i},
$$

where the semicolon indicates covariant differentiation with respect to the metric $\sigma_{i j}\left(t_{0}, x\right)$ and where $\hat{h}_{a b}$ is the second fundamental form of $\partial B_{R}$ with respect to the outward normal. Note that

$$
u_{; i j}=u_{: i j}+c_{i j}|D u|^{2},
$$

where $c_{i j}$ is a bounded tensor. Choosing now the coordinates $\left(\xi^{a}\right)$ at $\xi_{0}$ such that

$$
x_{a}^{i}=\delta_{a}^{i}
$$

we conclude

$$
-\sum_{i=1}^{n-1} \sigma^{i j} u_{; i j}=\mathscr{H}|D u|^{2}+c|D u|^{2},
$$

in view of (4.57), (4.66) and (4.71).

Combining now the relations (4.72) and (4.65) we obtain in $y_{0}$

(4.73) $u_{; i j} \hat{\nu}^{i} \hat{\nu}^{j}\left(1+\tilde{v}^{2}|D u|^{2}\right)=-v \tilde{H}+v^{2}\left(\bar{H}+\bar{h}_{i j} \check{u}^{i} \check{u}^{j} \tilde{v}^{2}\right)+\mathscr{H}|D u|^{2}+c|D u|^{2}$

from which we immediately infer the estimate (4.53), in view of (4.59), (4.64) and the assumption that

$$
|D u|^{2} \geq \frac{1}{2}
$$


Let us also recall that

$$
v=\tilde{v}^{-1}
$$

\section{Existence of A solution of the Dirichlet Problem}

in this section we want to prove that the Dirichlet problem (3.1) on page 9 has a solution of class $C^{3, \alpha}$ where we assume that the function $f=f\left(x^{0}, x\right)$ is of class $C_{\mathrm{loc}}^{1, \alpha}(N)$ and satisfies the estimate

$$
H_{-}<\inf _{N} f \leq \sup _{N} f<H_{+} .
$$

Let

$$
M_{i}=\operatorname{graph} \psi_{i}, \quad i=1,2,
$$

be barriers satisfying

$$
\begin{gathered}
\psi_{1}<\psi_{2}, \\
H_{2}=H_{\left.\right|_{M_{2}}}>\sup f, \\
\inf \psi_{2}>t_{0},
\end{gathered}
$$

resp.

$$
H_{1}=H_{\left.\right|_{M_{1}}}<\inf f
$$

and

$$
\sup \psi_{1}<t_{0} \text {. }
$$

Then, we pick two constants $m_{i} \in I$ such that

$$
m_{1}<\inf \psi_{1}<\sup \psi_{2}<m_{2}
$$

and $\epsilon>0$ so small such that

$$
m_{2}+\epsilon \in I
$$

and

$$
m_{1}-\epsilon \in I \text {. }
$$

Furthermore, let $\beta_{i}$ be smooth real functions satisfying

$$
\beta_{1}(t)=\left\{\begin{aligned}
-1 & , t \leq-\epsilon, \\
0 & , t \geq 0,
\end{aligned}\right.
$$

and

$$
\beta_{2}(t)= \begin{cases}0 & , t \leq 0 \\ 1 & , t \geq \epsilon\end{cases}
$$

as well as

$$
\beta_{i}^{\prime} \geq 0
$$


Then, we look at the Dirichlet problem for $M=\operatorname{graph} u$ in $B_{R}$

$$
\begin{aligned}
H_{\left.\right|_{M}} & =f(x, u)-\tilde{\mu} \beta_{1}\left(u-m_{1}\right)-\tilde{\mu} \beta_{2}\left(u-m_{2}\right), \\
u_{\left.\right|_{\partial B_{R}}} & =t_{0},
\end{aligned}
$$

where $\tilde{\mu}>0$ is a sufficiently large constant. The exact value will be determined later. It will depend on $f$ and on geometric quantities of the ambient space in the capped region

$$
\mathscr{S}\left(m_{1}-\epsilon, m_{2}+\epsilon\right)=\left[m_{1}-\epsilon, m_{2}+\epsilon\right] \times \mathcal{S}_{0} .
$$

We shall prove that the Dirichlet problem (5.14) has a solution

$$
u \in C^{1, \alpha}\left(\bar{B}_{R}\right)
$$

satisfying the estimates

$$
m_{1}-\epsilon \leq u \leq m_{2}+\epsilon
$$

and

$$
\tilde{v} \leq c,
$$

where $c$ depends on $\tilde{\mu}$ but not on $\epsilon$ and $\beta_{i}^{\prime}$.

Then, if we would $\epsilon$ letting tend to zero, we would obtain a solution of a variational inequality, where the obstacles are given by the slices

$$
\left\{x^{0}=m_{i}\right\} \text {. }
$$

However, we do not need to do this, since, by employing the barriers and the comparison theorem, Theorem 3.3 on page 13, we shall show that the penalization terms $\beta_{i}$ vanish. But we shall later refer to the above argument, when we claim that a given variational inequality has a solution.

Assuming for the moment that the Dirichlet problem (5.14) has a solution we are then able to prove:

Theorem 5.1. The solution of the Dirichlet problem (5.14) actually satisfies the equation

$$
H_{\left.\right|_{M}}=f(u, x),
$$

since $u$ lies between the barriers $\psi_{i}$ in $(5.2)$

$$
m_{1}<\psi_{1}<u<\psi_{2}<m_{2}
$$

and hence the penalization terms vanish.

Proof. We shall only prove the upper estimate, since the argument for the lower estimate is similar, or even identical by simply switching the light-cone. Also note that proving the weaker inequality

$$
\psi \leq u \leq \psi_{2}
$$

is as good as the stronger inequality, since the $\beta_{i}$ terms would still vanish and the maximum principle would yield the final result. 
Thus, let us assume that

$$
\left\{x \in B_{R}: \psi_{2}(x)<u(x)\right\} \neq \emptyset .
$$

From the proof of Theorem 3.3 on page 13 we then conclude that there exists a maximizing future directed geodesic $\gamma=\left(\gamma^{\alpha}\right)$ from a point $p_{2} \in M_{2}$ to a point $p_{1} \in M$. Let $p_{1}$ have the coordinates $\left(u\left(x_{1}\right), x_{1}\right)$ and similarly express $p_{2}$ as $\left(\psi_{2}\left(x_{2}\right), x_{2}\right)$. Then we deduce

$$
u\left(x_{1}\right)>\psi_{2}\left(x_{2}\right)>\psi_{1}\left(x_{2}\right)>m_{1}
$$

and this estimate is also valid in a small ball $B_{\rho}\left(x_{1}\right)$, hence we have

$$
u-m_{1}>0 \quad \text { in } B_{\rho}\left(x_{1}\right)
$$

and, in view of the definition of $\beta_{1}$, we infer

$$
\beta_{1}\left(u-m_{1}\right)=0,
$$

hence,

$$
H_{\left.\right|_{M}}=f(u, x)-\tilde{\mu} \beta_{2}\left(u-m_{2}\right) \leq f(u, x)
$$

in $B_{\rho}\left(x_{1}\right)$ and the further arguments in the proof of Theorem 3.3 lead to a contradiction.

Let us now prove that the Dirichlet problem (5.14) has a solution $u \in$ $C^{3, \alpha}\left(\bar{B}_{R}\right)$. We shall argue similarly as in the proof of [4, Theorem 5.2], where we treated a related problem. Since we then considered compact hypersurfaces without boundary and also used a different method for the gradient estimate, we cannot simply refer to that result but have to argue a little more detailed.

For the existence proof we shall use a Leray-Schauder type fixed point argument. For technical reasons it is therefore necessary to consider $M$ embedded in the conformal space $\left(\tilde{N}, \tilde{g}_{\alpha \beta}\right)$, where $\bar{g}_{\alpha \beta}$ and $\tilde{g}_{\alpha \beta}$ are related by

$$
\bar{g}_{\alpha \beta}=e^{2 \psi} \tilde{g}_{\alpha \beta} \text {. }
$$

We already used this embedding of $M$ in the proof of Lemma 4.8 on page 20 . Now, we need a more detailed description how the geometric quantities of the two embeddings are related. As before we embellish the geometric symbols in $\tilde{N}$ by a tilde. The geometric quantities are related by

$$
\begin{gathered}
g_{i j}=e^{2 \psi} \tilde{g}_{i j}, \\
\nu=e^{-\psi} \tilde{\nu}, \\
h_{i j} e^{-\psi}=\tilde{h}_{i j}+\psi_{\alpha} \tilde{\nu}^{\alpha} \tilde{g}_{i j}, \\
e^{\psi} H=\tilde{H}+n \psi_{\alpha} \tilde{\nu}^{\alpha},
\end{gathered}
$$

cf. [7, Proposition 1.1.11].

Let $u_{i j}$ be the covariant second derivatives of $u$ with respect to $\tilde{g}_{i j}$, then

$$
u_{i j}=\tilde{v}^{2} u_{; i j},
$$


where the covariant derivatives on the right-hand side are with respect to the metric $\sigma_{i j}(u, x)$, cf. [7, Lemma 2.7.6], and hence

$$
\tilde{h}_{i j} \tilde{v}=-\tilde{v}^{2} u_{i i j}+\bar{h}_{i j},
$$

where $\bar{h}_{i j}$ is the second fundamental form of the coordinate slices

$$
\left\{x^{0}=\text { const }\right\}
$$

in $\tilde{N}$-here, we refrain to embellish the barred quantities by an additional tilde - and we further deduce

$$
\tilde{H} \tilde{v}=-\tilde{v}^{2} \tilde{g}^{i j} u_{; i j}+\bar{H}+\tilde{v}^{2} \bar{h}_{i j} \check{u}^{i} \check{u}^{j},
$$

where we recall

$$
\check{u}^{i}=\sigma^{i j} u_{j} .
$$

Thus, the Dirichlet problem (5.14) is equivalent to

$$
\begin{aligned}
\tilde{H}_{\left.\right|_{M}} & =e^{\psi}\left\{f(u, x)-\tilde{\mu} \beta_{1}\left(u-m_{1}\right)-\tilde{\mu} \beta_{2}\left(u-m_{2}\right)+n D_{\alpha}\left(e^{-\psi}\right) \tilde{\nu}^{\alpha}\right\}, \\
u_{\left.\right|_{\partial B_{R}}} & =t_{0} .
\end{aligned}
$$

Let us also note that

$$
\tilde{v} \tilde{g}^{i j} u_{; i j}=D_{i}\left(\tilde{v} \sigma^{i j} u_{j}\right)
$$

Furthermore, by shifting the time coordinate we may assume $0 \in I$ and

$$
t_{0}=0 .
$$

The partial differential equation in (5.38) can be expressed in the form

$$
\begin{gathered}
-a^{i j} u_{; i j}+\tilde{v} \bar{h}_{i j} \check{u}^{i} \breve{u}^{j}= \\
e^{\psi}\left\{f(u, x)-\tilde{\mu} \beta_{1}\left(u-m_{1}\right)-\tilde{\mu} \beta_{2}\left(u-m_{2}\right)+n \alpha\left(e^{-\psi}\right)_{\alpha} \tilde{\nu}^{\alpha}\right\} \\
-v \bar{H}(u, x)
\end{gathered}
$$

or, if we abbreviate the right-hand side of the equation above by

$$
\tilde{f}=\tilde{f}(x, u, D u),
$$

in the form

$$
-a^{i j} u_{; i j}+\tilde{v} \bar{h}_{i j} \check{u}^{i} \check{u}^{j}-\tilde{f}=0 .
$$

Here, the tensor $a^{i j}$ is defined by

$$
a^{i j}=\tilde{v} \tilde{g}^{i j} .
$$

Let $a^{i}$ be the vector field

$$
a^{i}(p)=\tilde{v} \sigma^{i j} p_{j},
$$

where

$$
\tilde{v}=\left(1-|p|^{2}\right)^{-1}
$$

and where we assume

$$
|p|^{2}=\sigma^{i j} p_{i} p_{j}<1,
$$


then

$$
a^{i j}=\frac{\partial a^{i}}{\partial p_{j}} .
$$

To be absolutely precise, we should write

$$
a^{i}=a^{i}\left(x, x^{0}, p\right)
$$

and correspondingly

$$
a^{i j}=a^{i j}\left(x, x^{0}, p\right) .
$$

In the equation (5.43) we consider

$$
a^{i j}=a^{i j}(x, u, D u) .
$$

Let $m_{i}^{\prime} \in I$ be constants satisfying

$$
m_{1}^{\prime}<m_{1}-\epsilon
$$

and

$$
m_{2}^{\prime}>m_{2}+\epsilon,
$$

then we want to solve the variational inequality

$$
\left\langle-a^{i j} u_{; i j}+\tilde{v} \bar{h}_{i j} \check{u}^{i} \check{u}^{j}-\tilde{f}, v-u\right\rangle \geq 0 \quad \forall v \in K,
$$

where the convex set $K$ is defined by

$$
K=\left\{v \in H^{2,2}\left(B_{R}\right) \cap H_{0}^{1,2}\left(B_{R}\right): m_{1}^{\prime} \leq v \leq m_{2}^{\prime}\right\}
$$

and $u \in K$ is supposed to satisfy the additional requirement

$$
\tilde{v}<\infty \text {. }
$$

The duality in (5.54) is the real $L^{2}$ scalar product.

Remark 5.2. Let us emphasize that for the definition of Sobolev or Hölder spaces we equip the underlying domains, here, $B_{R}$, with a metric $\sigma_{i j}\left(x^{0}, x\right)$ with a constant $x^{0}$. In the present case we choose $x^{0}=0$. Note that the metrics $\sigma_{i j}(0, x)$ and $\sigma_{i j}\left(x^{0}, x\right)$ are all equivalent as long as $x^{0}$ stays in a compact subset of $I$.

To find a solution of (5.54) we shall apply a version of the Leray-Schauder fixed point theorem. Consider in $C_{0}^{1, \alpha}\left(\bar{B}_{R}\right)$ the closed set

$$
\mathscr{C}=\left\{w \in C^{1, \alpha}\left(\bar{B}_{R}\right): m_{1}^{\prime} \leq w \leq m_{2}^{\prime},|D w|^{2} \leq 1-\theta,|w|_{1, \alpha} \leq c\right\},
$$

where

$$
|D w|^{2}=\sigma^{i j}(w, x) w_{i} w_{j},
$$

and

$$
C_{0}^{1, \alpha}\left(\bar{B}_{R}\right)=\left\{w \in C^{1, \alpha}\left(\bar{B}_{R}\right): w_{\left.\right|_{\partial B_{R}}}=0\right\} .
$$

Here, $0<\alpha<1, \theta<1$ and $c$ are positive constants to be determined later. 
$\mathscr{C}$ is closed with non-empty interior, since

$$
0 \in \stackrel{\circ}{\mathscr{C}} \text {. }
$$

For $w \in \mathscr{C}$ consider the differential operator

$$
L u=-a^{i j} D_{i} D_{j} u+\tilde{v} \bar{h}_{i j} \check{w}^{i} \check{u}^{j}-\tilde{f},
$$

where

$$
\begin{gathered}
a^{i j}=a^{i j}(x, w, D w), \\
\tilde{v}=\left(1-|D w|^{2}\right)^{-1}, \\
\sigma_{i j}=\sigma_{i j}(w, x)
\end{gathered}
$$

and

$$
\tilde{f}=\tilde{f}(x, w, D w) .
$$

The covariant differentiation is with respect to $\sigma_{i j}$. Define the map

$$
T: \mathscr{C} \rightarrow C_{0}^{1, \alpha}\left(\bar{B}_{R}\right)
$$

by the requirement that

$$
u=T w
$$

is a solution of the variational inequality

$$
\langle L u-\tilde{f}, v-u\rangle \geq 0 \quad \forall v \in K .
$$

It is well-known that this variational inequality has a unique solution satisfying

$$
L u \in L^{\infty}\left(B_{R}\right)
$$

Hence, we have

$$
u \in H^{2, p}\left(B_{R}\right) \quad \forall 1<p<\infty,
$$

because of the Calderon-Zygmund inequalities. The existence of a solution of the variational inequality (5.68) and the relation (5.69) can be proved simultaneously with the help of the penalization method, i.e., by looking at Dirichlet problems similar to (5.14), where in case of linear uniformly elliptic equations the Schauder theory guarantees the solvability of Dirichlet problems assuming appropriate smoothness of the data. A solution of the variational inequality can be achieved by letting the parameter $\epsilon$, which enters in the definition of the penalization functions, tend to zero.

If we choose the exponent $p$ in (5.70) large enough such that

$$
\beta=1-\frac{n}{p}>\alpha
$$

then the embedding

$$
H^{2, p}\left(B_{R}\right) \hookrightarrow C^{1, \alpha}\left(\bar{B}_{r}\right)
$$

is compact, hence $T$ is compact. 
Now, let $u \in \mathscr{C}$ be an arbitrary quasi fixed point, i.e., there exists $\tilde{\lambda}>1$ such that

$$
T u=\tilde{\lambda} u \text {. }
$$

If we can show that

$$
u \in \stackrel{\circ}{\mathscr{C}}
$$

then $T$ will have a fixed point, cf. [11, Theorem 4.43], and it will be a solution of the variational inequality (5.54).

Thus, let $u$ be the quasi fixed point in (5.73). In the open set

$$
\Omega=\left\{x \in B_{r}: m_{1}^{\prime}<u(x)<m_{2}^{\prime}\right\}
$$

it will solve the equation

$$
-a^{i j} D_{i} D_{j} u+\tilde{v} \bar{h}_{i j} \check{u}^{i} \check{u}^{j}=\tilde{\lambda}^{-1} \tilde{f}(x, u, D u) .
$$

Adding on both sides

$$
\tilde{v}^{-1} \bar{H}(x, u)
$$

we deduce, in view of (5.36), that

$$
\tilde{H}=\tilde{\lambda}^{-1} \tilde{f}(x, u, D u)+\tilde{v}^{-1} \bar{H} \quad \text { in } \Omega,
$$

where $\tilde{H}$ is the mean curvature of $M=\operatorname{graph} u$ in $\tilde{N}$. We note that $\Omega$ reaches up to the boundary because

$$
m_{1}^{\prime}<0<m_{2}^{\prime}
$$

in view of (5.21) and the definition of the $m_{i}^{\prime}$ in (5.52)and (5.53), and that

$$
\left\{x \in B_{R}: D u \neq 0\right\} \subset \Omega \text {. }
$$

We also emphasize that $u$, which is already of class $H^{2, p}\left(B_{R}\right)$, cf. (5.70), satisfies

$$
u \in C^{3, \alpha}\left(\Omega \cup \partial B_{R}\right),
$$

because of the Schauder estimates, provided $\partial B_{R}$ is of class $C^{3, \alpha}$ which we assume.

By definition $u$ is bounded by the constants $m_{i}^{\prime}$ and hence we can try to apply the a priori estimates in Theorem 4.1 on page 14 in the present situation. We only have to check that the additional terms on the right-hand side of (5.78) do not alter the structure of the inequality (4.20) on page 16 and especially that the values of $\tilde{\lambda}$ and $\beta_{i}^{\prime}$ do not enter into the estimates. Recall that $\tilde{f}$ is defined to be the right-hand side of the equation (5.41).

First, we observe that when we estimate $\tilde{v}$, or better,

$$
w=\tilde{v} e^{\mu e^{\lambda(u+c)}}
$$

we assume

$$
\tilde{v}>2
$$


i.e., we consider points in $\Omega$ for the interior estimates or points in $\partial B_{R}$ for the boundary estimates but never points where $D u=0$.

In case of the boundary estimates we already switched to the conformal embedding so that the additional terms coming from that embedding are already taken care of, and from the original mean curvature $H$ only the supremum norm of $H$ will enter, hence only $\tilde{\mu}$ will enter into the estimates which is fine.

Thus, we only have to consider the interior estimates, where now the ambient metric is $\tilde{g}_{\alpha \beta}$. Let us look at the right hand side of (4.11) on page 15, where now

$$
\left(\eta_{\alpha}\right)=(-1,0, \ldots, 0) .
$$

The crucial term is the last one involving $f$ which is the prescribed mean curvature. In the present situation $f$ has to be replaced by

$$
\tilde{H}=\tilde{\lambda}^{-1} \tilde{f}+\tilde{v}^{-1} \bar{H} .
$$

The crucial term on the right hand-side of (4.11) has the form

$$
-\tilde{H}_{\beta} x_{i}^{\beta} x_{k}^{\alpha} \tilde{g}^{i k} \eta_{\alpha}=\tilde{H}_{i} u^{i}
$$

where

$$
u^{i}=\tilde{g}^{i j} u_{j} .
$$

From (5.85) we conclude that we only have to worry about

$$
\tilde{\lambda}^{-1} \tilde{f}_{i} u^{i}
$$

and hence, in view of (5.41) and (5.42), only about

$$
-\tilde{\lambda}^{-1} \tilde{\mu}\left(\beta_{1}^{\prime}+\beta_{2}^{\prime}\right)\|D u\|^{2} \leq 0
$$

which is non-positive and can be ignored, and in addition about the term

$$
-n \tilde{\lambda}^{-1} \psi_{\alpha \beta} \tilde{\nu}^{\alpha} x_{i}^{\beta} u^{i}-n \tilde{\lambda}^{-1} \psi_{\alpha} x_{k}^{\alpha} \tilde{h}_{i}^{k} u^{i},
$$

which can be estimated from above by

$$
c \tilde{v}^{3}+c\|\tilde{A}\|^{2}\|D u\|^{2} \leq c \tilde{v}^{3}+\epsilon\|\tilde{A}\|^{2} \tilde{v}+c \epsilon^{-1} \tilde{v}^{3},
$$

where the $c$ on the right-hand side of the inequality is slightly larger than the $c$ on the left-hand side, and hence satisfies the structural requirements of the right-hand side of (4.20) on page 16. Here, we also used

$$
\tilde{\lambda}>1 \text {. }
$$

Let us formulate the final conclusion as a lemma:

Lemma 5.3. Let $u$ be a quasi solution of $T$, then

$$
\sigma^{i j} u_{i} u_{j} \leq 1-\theta_{0}<1,
$$

where $\theta_{0}$ depends on the quantities mentioned in Theorem 4.1 on page 14 and furthermore on $\tilde{\mu}$ but not on $\tilde{\lambda}$ or $\beta_{i}^{\prime}$. We also emphasize that $|u|$ is bounded by $\max \left(m_{2}^{\prime},-m_{1}^{\prime}\right)$ which can be made arbitrarily close to $\max \left(m_{2}+\epsilon,-m_{1}-\epsilon\right)$. 
Next, let us show that the $C^{1, \alpha}$-norm of $u$ is a priori bounded.

Lemma 5.4. Let $u$ be a quasi fixed point of $T$, then

$$
|u|_{1, \alpha} \leq c_{1},
$$

where $c_{1}$ depends on $\theta_{0},|\tilde{f}|_{0}, R, \max \left(m_{2},-m_{1}\right)$ and the $C^{2}$-norm of $\partial B_{R}$ but is independent of the constants defining $\mathscr{C}$.

Proof. Since we know that now $a^{i j}$ is uniformly positive definite, let us write

$$
-a^{i j} u_{; i j}=-D_{i}\left(\tilde{v} \sigma^{i j} D_{j} u\right)=-D_{i}\left(a^{i}(x, u, D u)\right),
$$

cf. (5.39), where the covariant derivative is with respect to $\sigma_{i j}(u, x)$. From (5.69) we then obtain

$$
-D_{i}\left(a^{i}(x, u, D u)\right)=f^{0} \quad \text { in } B_{R},
$$

where

$$
f^{0} \in L^{\infty}\left(B_{R}\right)
$$

and that

$$
u_{\left.\right|_{\partial B_{R}}}=0 .
$$

We know that $u$ is Lipschitz continuous, $\partial B_{R}$ of class $C^{2}$ and the vector field $a^{i}\left(x, x^{0}, p\right)$ of class $C^{1}$ in its arguments, at least for allowed values of $\left(x^{0}, p\right)$ and

$$
a^{i j}=\frac{\partial a^{i}}{\partial p_{j}}
$$

is uniformly elliptic for the allowed values. Hence it is known that

$$
u \in H^{2, p}\left(B_{R}\right) \quad \forall 1<p<\infty
$$

and

$$
\|u\|_{2, p} \leq c_{2},
$$

where $c_{2}$ depends on $p, R$, and known constants, for details see the appendix in Section 9 on page 58.

Especially we have

$$
|u|_{1, \alpha} \leq c_{1},
$$

where $c_{1}$ is independent of the constants defining $\mathscr{C}$ only depending on the quantities mentioned in Lemma 5.4.

It finally remains to prove that a quasi fixed point of $T$ does not touch the obstacles.

Lemma 5.5. Let $u$ be a quasi fixed point of $T$, then $u$ satisfies the inequalities

$$
m_{1}^{\prime}<u<m_{2}^{\prime},
$$

and hence, $u$ also satisfies the equation (5.76) in $B_{R}$. 
Proof. We shall only prove the first inequality, since the proof of the other inequality is similar.

Let $x \in B_{R}$ be a point where

$$
u\left(x_{1}\right)=m_{1}^{\prime},
$$

then $u$ attains its minimum in $x_{1}$ and we have

$$
D u\left(x_{1}\right)=0 .
$$

Moreover,

$$
u<m_{2}^{\prime} \quad \text { in } B_{\rho}\left(x_{1}\right)
$$

for small $\rho$, hence we infer that in $B_{\rho}\left(x_{1}\right)$

$$
\begin{aligned}
& -a^{i j} D_{i} D_{j} u+\tilde{v} \bar{h}_{i j} \check{u}^{i} \check{u}^{j} \geq \tilde{\lambda}^{-1} \tilde{f}(x, u, D u) \\
& =\tilde{\lambda}^{-1} e^{\psi}\left\{f(u, x)-\tilde{\mu} \beta_{1}\left(u-m_{1}\right)-n \psi_{\alpha} \tilde{\nu}^{\alpha}-v \bar{H}(u, x)\right\} .
\end{aligned}
$$

In $x_{1}$ we have

$$
u-m_{1}=m_{1}^{\prime}-m_{1}<-\epsilon,
$$

hence, this inequality will also be valid in $B_{\rho}\left(x_{1}\right)$ if $\rho$ is small and we further deduce

$$
\beta_{1}\left(u-m_{1}\right)=-1
$$

in $B_{\rho}\left(x_{1}\right)$. Thus, we conclude that in $x_{1}$

$$
f(u, x)+\tilde{\mu}+n \dot{\psi}-\bar{H}(u, x)>0,
$$

if $\tilde{\mu}$ is large enough, which we stipulate. Since $u$ is of class $C^{1, \alpha}$ the righthand side of (5.107) will therefore be strictly positive if $\rho$ is small for the same $\tilde{\mu}$ as in (5.110). But this leads to a contradiction when we apply the weak Harnack inequality to $\left(u-m_{1}^{\prime}\right)$. Indeed, the left-hand side of $(5.107)$ can be written in the form

$$
-D_{i}\left(\tilde{v} \sigma^{i j} u_{j}\right)+\tilde{v} \bar{h}_{i j} \check{u}^{i} \check{u}^{j}
$$

and this uniformly elliptic divergence operator should be strictly positive in $B_{\rho}\left(x_{1}\right)$. But then $\left(u-m_{1}^{\prime}\right)$, which is non-negative, cannot attain a minimum in $B_{\rho}\left(x_{1}\right)$ contrary to our assumption.

Thus, we have proved that $T$ has a fixed point which is actually a solution of the Dirichlet problem (5.14) on page 23, and hence, also a solution of the original Dirichlet problem (3.1) on page 9, in view of Theorem 5.1. 


\section{Decay estimates For the SOLUtions}

In this section we want to derive decay estimates for solutions of the Dirichlet problems in (3.1) on page 9, where, now and for the rest of the paper, we assume for simplicity that

$$
f\left(x^{0}, x\right)=\hat{H}(t) \quad t \in I,
$$

i.e., in case of the Dirichlet problem (3.1)

$$
f=\hat{H}\left(t_{0}\right),
$$

for otherwise we would have to impose appropriate conditions on $f . \hat{H}(t)$ is the mean curvature of the slice

$$
\left\{x^{0}=t\right\}
$$

in the Robertson-Walker spacetime $\hat{N}$. The decay parameter is $r$, the radial distance function; $r$ is the geodesic distance from a fixed point of the underlying space of constant curvature, i.e., $\mathbb{R}^{n}$ or $\mathbb{H}^{n}$, where, to be absolutely precise, the metric of these spaces is multiplied by the scale factor $a^{2}\left(x^{0}\right)$, and hence, the corresponding distance $r$ also depends on $a\left(x^{0}\right)$

$$
r=a\left(x^{0}\right) \rho,
$$

where $\rho$ is the geodesic distance corresponding to $a=1$.

For the decay estimates we use the radial function in (6.4) with

$$
x^{0}=t_{0},
$$

the boundary values of the corresponding Dirichlet problem. Note, that the balls

$$
B_{R} \subset \mathcal{S}_{0}
$$

are defined with respect to $\rho$ in order to have a common domain of definition in case we want to compare solutions with different data.

For simplicity we shall redefine the balls for the decay estimates by assuming that the radius $R$ is defined by the geodesic distance

$$
r=a\left(t_{0}\right) \rho .
$$

We then look at the Dirichlet problem (3.1) in balls with $R>R_{0}$, where $R_{0}$ is so large such that the asymptotical estimates in Assumption 2.4 on page 7 are valid, especially the estimate in (2.52), namely,

$$
\dot{\bar{H}} \geq c_{0}>0
$$

uniformly in

$$
\left\{\left(x^{0}, x\right): r(x) \geq R_{0}, m_{1} \leq x^{0} \leq m_{2}\right\},
$$

where the $m_{i}, i=1,2$, are bounds for the solutions $u$ of the Dirichlet problems (3.1) which are independent of $R$.

Then can prove: 
Lemma 6.1. Let $u$ be a solution of the Dirichlet problem (3.1), then there exists $R_{1}>R_{0}$ such that $\left|u-t_{0}\right|$ can be estimated by

$$
\left|u(x)-t_{0}\right| \leq \frac{c}{r} \quad \forall x \in \bar{B}_{R} \cap\left\{r(x) \geq R_{1}\right\},
$$

where $R_{1}>R_{0}$ and the constants $R_{1}$ and $c$ depend on $c_{0}$ in (6.8) and other already known estimates for $|D u|$ and $|u|$ as well as the ambient capped region $\mathscr{S}\left(m_{1}, m_{2}\right)$.

Proof. We shall construct appropriate barrier functions to estimate $u$ from above and from below. Let $\varphi=\varphi(r)$ be defined by

$$
\varphi(r)=\frac{\lambda R_{1}}{r}+t_{0}
$$

be the upper barrier and

$$
\tilde{\varphi}(r)=-\frac{\lambda R_{1}}{r}+t_{0}
$$

be the lower barrier, where $\lambda>0$ is a fixed constant satisfying

$$
\lambda>\sup |u|+\left|t_{0}\right| \text {. }
$$

The value of $R_{1}$ will be determined later. Since the arguments for the upper resp. lower estimates are similar we shall only prove the upper estimate

$$
u(x) \leq \frac{\lambda R_{1}}{r}+t_{0} \quad \forall x \in B_{R} \cap\left\{r(x) \geq R_{1}\right\} .
$$

Let $\Omega$ be the region

$$
\Omega=\left\{x \in B_{R}: R_{1}<r(x)<R\right\},
$$

then we to prove the estimate $(6.14)$ in $\Omega$. The boundary of $\Omega$ consists of the spheres $\left\{r(x)=R_{1}\right\}$ and $\{r(x)=R\}$. On both spheres the inequality (6.14) is strict. Thus, let us argue by contradiction and assume that there exists $x_{0} \in \Omega$ such that

$$
u\left(x_{0}\right)-\varphi\left(x_{0}\right)=\sup _{\Omega}(u-\varphi)>0 .
$$

Then we know that in $x_{0}$

$$
D u=D \varphi
$$

and

$$
u_{, i j} \leq \varphi_{, i j},
$$

where the comma indicates partial derivatives. In order to apply the maximum principle we again consider $M$ to be embedded in $\left(\tilde{N}, \tilde{g}_{\alpha \beta}\right)$, then $u$ satisfies the elliptic equation

$$
\tilde{H} \tilde{v}=-\tilde{v}^{2} \tilde{g}^{i j} u_{; i j}+\tilde{\bar{H}}+\tilde{v}^{2} \tilde{\bar{h}}_{i j} \check{u}^{i} \check{u}^{j},
$$

where $\tilde{\bar{h}}_{i j}$ is the second fundamental form of the slices

$$
\left\{x^{0}=\text { const }\right\}
$$


in $\tilde{N}$, cf. (5.36) on page 25. The geometric quantities are related to the corresponding geometric quantities in $N$ by

$$
e^{\psi} H=\tilde{H}+n \psi_{\alpha} \tilde{\nu}^{\alpha}
$$

and

$$
e^{\psi} \bar{H}=\tilde{\bar{H}}-n \dot{\psi}
$$

Furthermore, we know that

$$
\left|e^{\psi}-1\right|+|\|D \psi\|| \leq \frac{c}{r} \quad \forall x \in\left\{r(x) \geq R_{0}\right\}
$$

and

$$
\tilde{v}-1=\frac{\tilde{v}^{2}}{\tilde{v}+1}|D u|^{2}
$$

Thus, we deduce

(6.25) $\hat{H}\left(t_{0}\right) \equiv f\left(t_{0}\right)=H \geq-\tilde{v}^{2} \tilde{g}^{i j} u_{; i j}+\bar{H}(u, x)-c|D u|^{2}-\frac{c}{r} \quad \forall x \in \Omega$,

where $\hat{H}\left(t_{0}\right)$ is the mean curvature of the slice

$$
\left\{x^{0}=t_{0}\right\}
$$

in the Robertson-Walker spacetime $\hat{N}$ and the identities on the left-hand side of the inequality simply reflect the prescribed value of $H$.

Next, let us consider the covariant derivatives of $u$. We have

$$
\begin{aligned}
-u_{; i j} & =-u_{, i j}+\Gamma_{i j}^{k} u_{k} \\
& \geq-\varphi_{, i j}+\hat{\Gamma}_{i j}^{k} \varphi_{k}+\left(\Gamma_{i j}^{k}-\hat{\Gamma}_{i j}^{k}\right) \varphi_{k},
\end{aligned}
$$

where $\hat{\Gamma}_{i j}^{k}$ are the Christoffel symbols of the metric $\hat{\sigma}_{i j}$ which is the induced metric of the slice

$$
\left\{x^{0}=t_{0}\right\}
$$

in $\hat{N}$. In view of the boundedness of $\tilde{v}$ and the assumptions (2.47) and (2.48) on page 8 we infer that the tensor

$$
\Gamma_{i j}^{k}-\hat{\Gamma}_{i j}^{k}
$$

is bounded relative to the metric $\sigma_{i j}$.

Moreover,

$$
\begin{gathered}
\varphi_{i}=-\frac{\lambda R_{1}}{r^{2}} r_{i}, \\
\varphi_{, i j}=2 \frac{\lambda R_{1}}{r^{3}} r_{i} r_{j}-\frac{\lambda R_{1}}{r^{2}} r_{, i j}
\end{gathered}
$$

and the tensor

$$
r_{, i j}-\hat{\Gamma}_{i j}^{k} r_{k}
$$


is uniformly bounded with respect to $\hat{\sigma}_{i j}$ and hence also with respect to $\sigma_{i j}$. Thus, we conclude from (6.25) and (6.27)

$$
\hat{H}\left(t_{0}\right) \geq \bar{H}(u, x)-\frac{c}{r}
$$

in $x=x_{0}$, where $c$ is independent of $R_{1}$. Furthermore, in view of (2.47) on page 8 ,

$$
\hat{H}\left(t_{0}\right) \leq \bar{H}\left(t_{0}, x\right)+\frac{c}{r} \quad \forall x \in \Omega
$$

and therefore

$$
\bar{H}\left(t_{0}, x_{0}\right) \geq \bar{H}(u, x)-\frac{c}{r},
$$

or equivalently,

$$
0 \geq \bar{H}(u, x)-\bar{H}\left(t_{0}, x_{0}\right)-\frac{c}{r},
$$

from which we conclude

$$
\begin{aligned}
0 & \geq \dot{\bar{H}}\left(\tau, x_{0}\right)\left(u-t_{0}\right)-\frac{c}{r} \\
& \geq c_{0} \frac{\lambda R_{1}}{r}-\frac{c}{r}>0,
\end{aligned}
$$

in view of $(2.52)$ on page 8 , provided

$$
R_{1}>\frac{c}{\lambda c_{0}}
$$

a contradiction.

The decay estimate for $\left|u-t_{0}\right|$ allows us to prove a similar decay estimate for $|D u|$ and $\left|D^{2} u\right|$, and also for higher derivatives, with the help of the Schauder estimates. But first, let us derive local Schauder estimates.

Lemma 6.2. Let $u$ be a solution of the Dirichlet problem (3.1) of class $C^{3, \alpha}$, then $u$ satisfies the elliptic differential equation

$$
-a^{i j} u_{; i j}+b^{i} u_{i}+c(x)\left(u-t_{0}\right)=\tilde{f},
$$

where the covariant differentiation is with respect to $\sigma_{i j}(u, x)$ and

$$
\begin{gathered}
a^{i j}=\tilde{v}^{2} \tilde{g}^{i j}, \\
b^{i}=\tilde{v}^{2} \dot{\psi} \sigma^{i j} u_{j}-n \tilde{v}^{2} \psi_{j} \sigma^{j i}-(n-1) \dot{\psi} \tilde{v}^{2} \sigma^{i j} u_{j} \\
+\tilde{v}^{2} e^{\psi} \bar{h}_{j k} \check{u}^{j} \sigma^{i k}-e^{\psi} \hat{H}\left(t_{0}\right) \frac{\tilde{v}^{2}}{\tilde{v}+1} \sigma^{j i} u_{j}, \\
c=e^{\psi} \int_{0}^{1} \dot{\bar{H}}\left(t u+(1-t) t_{0}, x\right) d t
\end{gathered}
$$

and

$$
\tilde{f}=e^{\psi}\left(\hat{H}\left(t_{0}\right)-\bar{H}\left(t_{0}, x\right)\right) .
$$


Then, for any $x_{0} \in B_{R}$ and any $\rho_{0}>0$ such that

$$
\bar{B}_{2 \rho_{0}}\left(x_{0}\right) \subset B_{R}
$$

the estimate

$$
\left|u-t_{0}\right|_{2, \alpha, B_{\rho_{0}}\left(x_{0}\right)} \leq c\left(|\tilde{f}|_{0, \alpha, B_{2 \rho_{0}}\left(x_{0}\right)}+\left|u-t_{0}\right|_{0, B_{2 \rho_{0}}\left(x_{0}\right)}\right)
$$

is valid, where $c$ depends on $\rho_{0}$ and known quantities but not on $R$.

Similarly, for any $x_{0} \in \partial B_{R}$ and any $\rho_{0}>0$ we have

$$
\left|u-t_{0}\right|_{2, \alpha, \Omega\left(x_{0}, \rho_{0}\right)} \leq c\left(|\tilde{f}|_{0, \alpha, \Omega\left(x_{0}, 2 \rho_{0}\right)}+\left|u-t_{0}\right|_{0, \Omega\left(x_{0}, 2 \rho_{0}\right)}\right),
$$

where $c$ depends on $\rho_{0}$ and known quantities but not on $R$. Here,

$$
\Omega\left(x_{0}, \rho_{0}\right)=B_{R} \cap B_{\rho_{0}}\left(x_{0}\right) .
$$

If the ambient space $N$ is of class $C^{m, \alpha}, m \geq 3,0<\alpha<1$, then

$$
\left|u-t_{0}\right|_{m, \alpha, B_{\rho_{0}}\left(x_{0}\right)} \leq c\left(|\tilde{f}|_{m-2, \alpha, B_{2 \rho_{0}}\left(x_{0}\right)}+\left|u-t_{0}\right|_{0, B_{2 \rho_{0}}\left(x_{0}\right)}\right)
$$

where $c$ depends on $\rho_{0}, m$, and known quantities but not on $R$, and near the boundary

$$
\left|u-t_{0}\right|_{m, \alpha, \Omega\left(x_{0}, \rho_{0}\right)} \leq c\left(|\tilde{f}|_{m-2, \alpha, \Omega\left(x_{0}, 2 \rho_{0}\right)}+\left|u-t_{0}\right|_{0, \Omega\left(x_{0}, 2 \rho_{0}\right)}\right)
$$

for any $x_{0} \in \partial B_{R}$ and $\rho_{0}>0$, where $c$ depends on $\rho_{0}, m$ and known quantities but not on $R$.

Proof. „(6.39)“ Follows immediately from (6.19) and (6.24).

„(6.45)" Since the coefficients in the operator in (6.39) are uniformly of class $C^{0, \alpha}$, cf. Remark 9.1 on page 60 , the estimate follows immediately from the interior Schauder estimates, cf. [9, Corollary 6.3].

„(6.46)" This estimate is due to the Schauder estimates near a $C^{2, \alpha}$ boundary having in mind that $\left(u-t_{0}\right)$ has zero boundary values, cf. $[9$, Corollary 6.7].

„(6.48)" Let us express the covariant derivatives in equation (6.39) as partial derivatives and let us differentiate this equation with respect to $x^{k}$, where $1 \leq k \leq n$ is fixed. The resulting equation is an elliptic equation for

$$
w=D_{k}\left(u-t_{0}\right) .
$$

Applying then the estimate (6.45) to $w$, while replacing $\rho_{0}$ by $\frac{\rho_{0}}{2}$, we deduce

$$
\begin{aligned}
|w|_{2, \alpha, B \frac{\rho_{0}}{2}\left(x_{0}\right)} & \leq c\left(|\tilde{f}|_{1, \alpha, B_{\rho_{0}}\left(x_{0}\right)}+\left|u-t_{0}\right|_{2, \alpha, B_{\rho_{0}}\left(x_{0}\right)}\right) \\
& \leq c\left(|\tilde{f}|_{1, \alpha, B_{2 \rho_{0}}\left(x_{0}\right)}+\left|u-t_{0}\right|_{0, B_{2 \rho_{0}}\left(x_{0}\right)}\right),
\end{aligned}
$$

in view of the previous result for $\left(u-t_{0}\right)$. Here the quotient of the radii is 4 instead of 2 , but it is obvious that any number larger than 1 would suffice.

Since $k$ is arbitrary, we have the estimate for $m=3$. The estimate for larger $m$ is then proved inductively having in mind that $\rho_{0}$ is flexible. 
„(6.49)" For the $C^{m, \alpha}$-estimates near the boundary we have to straighten the boundary either explicitly, or implicitly, by using geodesic polar coordinates $\left(r, \xi^{a}\right), 1 \leq a \leq n-1$, with respect to the induced metric of the slice

$$
\left\{x^{0}=t_{0}\right\}
$$

in $\hat{N}$. This coordinate system is also an allowed coordinate system if the slice is embedded in $N$ though not a geodesic polar coordinate system. But the coordinates $\left(R, \xi^{a}\right)$ describe the boundary $\partial B_{R}$ which is all we need. The partial derivatives with respect $\xi^{a}$ are then tangential derivatives and the function

$$
w=D_{a}\left(u-t_{0}\right)
$$

has zero boundary values. Similarly as in (6.46) we obtain, for any $x_{0} \in B_{R}$ and any $0<\rho_{0}<\frac{R}{4}$,

$$
\begin{aligned}
|w|_{2, \alpha, \Omega\left(x_{0}, \frac{\rho_{0}}{2}\right)} & \leq c\left(|\tilde{f}|_{1, \alpha, \Omega\left(x_{0}, \rho_{0}\right)}+\left|u-t_{0}\right|_{2, \alpha, \Omega\left(x_{0}, \rho_{0}\right)}\right) \\
& \leq c\left(|\tilde{f}|_{1, \alpha, \Omega\left(x_{0}, 2 \rho_{0}\right)}+\left|u-t_{0}\right|_{0, \Omega\left(x_{0}, 2 \rho_{0}\right)}\right),
\end{aligned}
$$

where $c$ is independent of $R$.

Let us denote the radial coordinate by $\xi^{n}$, then, in (6.54), we have estimated

$$
\sum_{i+j+k<3 n}\left|D_{i} D_{j} D_{k}\left(u-t_{0}\right)\right|_{0, \alpha, \Omega\left(x_{0}, \frac{\rho_{0}}{2}\right)} .
$$

The remaining derivative

$$
D_{n} D_{n} D_{n} u
$$

can be estimated by looking at the uniformly elliptic equation for

$$
w=D_{n} u
$$

and applying the estimate for (6.55) as well as the uniform positivity of $a^{n n}$.

The $C^{m, \alpha}$-estimates near the boundary for $m>3$ can then be proved inductively.

Combining the results of Lemma 6.1, Lemma 6.2 and the decay assumptions for $\left(e^{\psi}-1\right)$ and its derivatives resp. $\left(\hat{H}\left(t_{0}\right)-\bar{H}\left(t_{0}, x\right)\right)$ and its spatial derivatives, cf. Assumption 2.4 on page 7 , we can state:

Theorem 6.3. Let $N$ be of class $C^{m, \alpha}, m \geq 3,0<\alpha<1$, then for any ball

$$
\bar{B}_{2 \rho_{0}}\left(x_{0}\right) \subset B_{R} \backslash B_{R_{1}}
$$

resp. boundary neighbourhood

$$
\Omega\left(x_{0}, 2 \rho_{0}\right) \subset \bar{B}_{R} \backslash B_{R_{1}}
$$

the decay estimates

$$
\left|u-t_{0}\right|_{m, \alpha, B_{\rho_{0}}\left(x_{0}\right)} \leq \frac{c}{r\left(x_{0}\right)}
$$


resp.

$$
\left|u-t_{0}\right|_{m, \alpha, \Omega\left(x_{0}, \rho_{0}\right)} \leq \frac{c}{r\left(x_{0}\right)}
$$

are valid, where $c$ depends on $m, \rho_{0}$ and known quantities but not on $R$. Especially we obtain

$$
\sum_{0 \leq|\gamma| \leq m}\left|D^{\gamma}\left(u-t_{0}\right)(x)\right| \leq \frac{c}{r(x)} \quad \forall x \in \bar{B}_{R} \backslash B_{R_{1}},
$$

where $c$ depends on $m$ but not on $x$ or $R$.

Proof. Obvious.

Corollary 6.4. Let $u=u_{R}$ be a solution of the Dirichlet problem (3.1), then there exists $c_{0}>0$ such that

$$
\left(|A|^{2}+\bar{R}_{\alpha \beta} \nu^{\alpha} \nu^{\beta}\right) \geq c_{0}>0 \quad \forall x \in\left\{r(x)>R_{1}\right\},
$$

if $R_{1}$ is large enough.

Proof. This is due to the estimate (6.62) which implies that the hypersurface

$$
M=\operatorname{graph} u
$$

converges to the slice

$$
M\left(t_{0}\right)=\left\{x^{0}=t_{0}\right\}
$$

in the class $C^{2}$ if $r(x)$ is large, i.e., the left-hand side of (6.63) uniformly converges to the corresponding quantity of $M\left(t_{0}\right)$ which satisfies this inequality because of $(2.52),(2.49)$ and the relation

$$
\dot{H}=-\Delta e^{\psi}+\left(|A|^{2}+\bar{R}_{\alpha \beta} \nu^{\alpha} \nu^{\beta}\right) e^{\psi}
$$

which is valid for the slices

$$
\left\{x^{0}=\text { const }\right\}
$$

cf. [7, equ. (2.3.37), p. 96].

\section{Existence of a CMC Foliation}

Let $u_{R}$ be a solution of the Dirichlet problems (3.1) on page 9 with boundary values $t_{0}$. In view of the estimates in the previous section we infer that, by letting $R$ tend to infinity, a subsequence converges in

$$
C_{\text {loc }}^{m, \alpha}\left(\mathcal{S}_{0}\right) \cap C_{\text {loc }}^{m}\left(\overline{\mathcal{S}}_{0}\right),
$$

where the subscript "loc" is necessary since the $u_{R}$ are only defined in $B_{R}$, to a function

$$
u \in C^{m, \alpha}\left(\mathcal{S}_{0}\right) \cap C^{m}\left(\overline{\mathcal{S}}_{0}\right)
$$

such that $M=$ graph $u$ is a spacelike hypersurface of constant mean curvature

$$
F_{\left.\right|_{M}}=f\left(t_{0}\right)=\hat{H}\left(t_{0}\right) \text {. }
$$


Here, the function space

$$
C^{m}\left(\overline{\mathcal{S}}_{0}\right) \subset C^{m}\left(\mathcal{S}_{0}\right)
$$

is defined by the additional requirements

$$
t=\lim _{r(x) \rightarrow \infty} u(x) \in I \quad \forall u \in C^{2}\left(\overline{\mathcal{S}}_{0}\right)
$$

and that

$$
|u(x)-t| \leq \frac{c}{r(x)} \quad \forall x \in\left\{r(x) \geq R_{1}\right\}
$$

as well as

$$
\sum_{0 \leq|\gamma| \leq m}\left|D^{\gamma}\left(u-t_{0}\right)(x)\right| \leq \frac{c}{r(x)} \quad \forall x \in\left\{r(x) \geq R_{1}\right\}
$$

for suitable large constants $c$ and $R_{1}$.

Let us write

$$
u=u\left(t_{0}, x\right)
$$

for the function in (7.2), where

$$
t_{0}=\lim _{r(x) \rightarrow \infty} u(x) .
$$

Since the mean curvature function

$$
\tau=\hat{H}(t) \quad t \in I
$$

is invertible, recall that

$$
\dot{\hat{H}}>0
$$

we could also express $u$ in the form

$$
u=u\left(\tau_{0}, x\right),
$$

though, at the moment, the convention (7.8) is more suitable. We shall also speak of the solution, since we shall later prove that the sequence $u_{R}$ actually converges and not only subsequences, because the solutions of equation (7.3) satisfying the estimate (7.6) are uniquely determined.

Moreover, in view of the Comparison Theorem 3.3 on page 13 we conclude that

$$
t_{1}<t_{2} \rightarrow u\left(t_{1}, x\right)<u\left(t_{2}, x\right) \quad \forall\left(t_{i}, x\right) \in I \times \mathcal{S}_{0} .
$$

The strict inequality on the right-hand side is again due to the weak Harnack inequality, cf. the proof of Lemma 7.2, where this argument is applied in a more detailed fashion.

The functions $u(t, \cdot)$ also satisfy: 
Lemma 7.1. Let

$$
M_{t}=\operatorname{graph} u(t, \cdot) \quad t \in I=\left(t_{-}, t_{+}\right),
$$

then

$$
\lim _{t \rightarrow t_{+}} u(t, x)=t_{+} \quad \forall x \in \mathcal{S}_{0}
$$

and

$$
\lim _{t \rightarrow t_{-}} u(t, x)=t_{-} \quad \forall x \in \mathcal{S}_{0} .
$$

Proof. We shall only prove the first relation. Let $M_{k}=$ graph $\psi_{k}$ be an arbitrary barrier such that

$$
H_{k}=H_{\left.\right|_{k}}
$$

and

$$
t_{k}=\sup \psi_{k},
$$

then, if $u_{R}(t, \cdot)$ is a solution of the Dirichlet problem in $B_{R}$ with boundary value $t$ and mean curvature $\hat{H}(t)$, we deduce

$$
u_{R}(t, x)>\psi_{k}(x) \quad \forall x \in B_{R}
$$

provided

$$
t>t_{k} \wedge \hat{H}(t)>H_{k},
$$

in view of Theorem 3.3 on page 13 . The requirements (7.20) can be easily satisfied because

$$
\lim _{t \rightarrow t_{+}} \hat{H}(t)=H_{+} .
$$

The estimate (7.19) is then also valid for $u(t, \cdot)$, provided (7.20) is true, from which the relation (7.15) can be easily deduced in view of the properties of the barriers.

Let us now show that the spacelike hypersurfaces over $\mathcal{S}_{0}$

$$
M=\operatorname{graph} u
$$

satisfying

$$
H_{\left.\right|_{M}}=c_{0}=\mathrm{const}
$$

and

$$
\left|u(x)-t_{0}\right| \leq \frac{c}{r(x)} \quad \forall x \in\left\{r(x)>R_{1}\right\}
$$

are uniquely determined.

Lemma 7.2. Let $M=$ graph $u$ be a spacelike hypersurface of class $C^{3, \alpha}\left(\mathcal{S}_{0}\right)$ satisfying $(7.23)$ and $(7.24)$, then $M$ is unique. 
Proof. We argue by contradiction by assuming there exists two hypersurfaces

$$
M_{i}=\operatorname{graph} u_{i}, \quad i=1,2,
$$

satisfying (7.23) as well as (7.24). Let us suppose that

$$
0<d_{0}=d\left(M_{2}, M_{2}\right),
$$

then there exists $p_{k} \in M_{2}$ and $q_{k} \in M_{1}$ such that

$$
d\left(p_{k}, q_{k}\right) \rightarrow d_{0} .
$$

The points can be expressed in the form

$$
p_{k}=\left(u_{2}\left(x_{k}\right), x_{k}\right)
$$

and similarly

$$
q_{k}=\left(u_{1}\left(y_{k}\right), y_{k}\right)
$$

If $r\left(x_{k}\right)$ remains uniformly bounded, then $r\left(y_{k}\right)$ is also uniformly bounded because

$$
q_{k} \in I^{+}\left(p_{k}\right),
$$

and vice versa. On the other hand, if $r\left(x_{k}\right)$ and $r\left(y_{k}\right)$ both tend to infinity, then for any $\epsilon>0$ there exists $k_{0}$ such that

$$
t_{0}-\epsilon<u_{1}\left(y_{k}\right)<t_{0}+\epsilon \quad \forall k \geq k_{0}
$$

$$
t_{0}-\epsilon<u_{2}\left(x_{k}\right)<t_{0}+\epsilon \quad \forall k \geq k_{0} .
$$

Let $\gamma_{k}$ be the future directed curve connecting $p_{k}$ and $q_{k}$, then $\gamma_{k}$ can be extended to a future directed curve $\tilde{\gamma}_{k}$ connecting the slices

$$
M\left(t_{0}-\epsilon\right)=\left\{x^{0}=t_{0}-\epsilon\right\}
$$

and

$$
M\left(t_{0}+\epsilon\right)=\left\{x^{0}=t_{0}+\epsilon\right\}
$$

such that the lengths of the extended curves satisfy

$$
L\left(\tilde{\gamma}_{k}\right)>L\left(\gamma_{k}\right) \geq \frac{d_{0}}{2} \quad \forall k \geq k_{0},
$$

if $k_{0}$ is large enough. But this is a contradiction since then

$$
\frac{d_{0}}{2} \leq d\left(M\left(t_{0}-\epsilon\right), M\left(t_{0}+\epsilon\right)\right) \equiv d_{\epsilon}
$$

and

$$
\lim _{\epsilon \rightarrow 0} d_{\epsilon}=0 .
$$

Hence, we conclude that there exist $p_{i}, i=1,2$,

$$
p_{i} \in M_{i}
$$

such that

$$
d_{0}=d\left(p_{2}, p_{1}\right)=d\left(M_{2}, M_{1}\right),
$$


where $d$ is the Lorentzian distance function. Let $\varphi$ be a maximal geodesic from $M_{2}$ to $M_{1}$ realizing this distance with endpoints $p_{2}$ and $p_{1}$, and parametrized by arc length.

Denote by $\bar{d}$ the Lorentzian distance function to $M_{2}$, i.e., for $p \in I^{+}\left(M_{2}\right)$

$$
\bar{d}(p)=\sup _{q \in M_{2}} d(q, p) .
$$

Since $\varphi$ is maximal, $\Gamma=\left\{\varphi(t): 0 \leq t<d_{0}\right\}$ contains no focal points of $M_{2}$, cf. [12, Theorem 34, p. 285], hence there exists an open neighbourhood $\mathcal{V}=\mathcal{V}(\Gamma)$ such that $\bar{d}$ is smooth in $\mathcal{V}$, cf. [12, Proposition 30], because $\bar{d}$ is a component of the inverse of the normal exponential map of $M_{2}$.

Now, $M_{2}$ is the level set $\{\bar{d}=0\}$, and the level sets

$$
M(t)=\{p \in \mathcal{V}: \bar{d}(p)=t\}
$$

are $C^{3}$ hypersurfaces; $x^{0}=\bar{d}$ is a time function in $\mathcal{V}$ and generates a normal Gaussian coordinate system, since $\langle D \bar{d}, D \bar{d}\rangle=-1$. Thus, the mean curvature $\bar{H}(t)$ of $M(t)$ satisfies the equation

$$
\dot{\bar{H}}=|\bar{A}|^{2}+\bar{R}_{\alpha \beta} \nu^{\alpha} \nu^{\beta},
$$

cf. [7, equ. (2.3.27, p. 96], and therefore we have

$$
\dot{\bar{H}} \geq 0,
$$

in view of the assumption (2.53).

Next, consider a local tubular neighbourhood $\mathcal{U}$ of $M_{1}$ near $p_{1}$-to simply the phrasing - with corresponding normal Gaussian coordinates $\left(x^{\alpha}\right)$. The (local) level sets

$$
\tilde{M}(s)=\left\{x^{0}=s\right\}, \quad-\epsilon<s<0,
$$

lie in the past of $M_{1}=\tilde{M}(0)$ and are of class $C^{3, \alpha}$ for small $\epsilon$.

Since the geodesic $\varphi$ is normal to $M_{1}$, it is also normal to $\tilde{M}(s)$ and the length of the geodesic segment of $\varphi$ from $\tilde{M}(s)$ to $M_{1}$ is exactly $-s$, i.e., equal to the distance from $\tilde{M}(s)$ to $M_{1}$, hence we deduce

$$
d\left(M_{2}, \tilde{M}(s)\right)=d_{0}+s,
$$

i.e., $\left\{\varphi(t): 0 \leq t \leq d_{0}+s\right\}$ is also a maximal geodesic from $M_{2}$ to $\tilde{M}(s)$, and we conclude further that, for fixed $s$, the hypersurface $\tilde{M}(s) \cap \mathcal{V}$ is contained in the past of $M\left(d_{0}+s\right)$ and touches $M\left(d_{0}+s\right)$ in $p_{s}=\varphi\left(d_{0}+s\right)$.

Let $p_{2}$ have the coordinates $\left(0, x_{0}\right)$ in the normal Gaussian coordinate system defined in the tubular neighbourhood $\mathcal{V}$, then there exists a small ball around $x_{0}$

$$
B_{\delta}\left(x_{0}\right) \subset M_{2}
$$

such that the cylinder

$$
Q=\left[0, d_{0}+s\right] \times B_{\delta}\left(x_{0}\right) \subset \mathcal{V}
$$


and the hypersurface

$$
\tilde{M}(s) \cap Q
$$

can be written as a graph over $B_{\delta}\left(x_{0}\right)$, where the time coordinate is $x^{0}=\bar{d}$. Note that

$$
\hat{M}(s)=\tilde{M}(s) \cap Q=\left\{\left(x^{0}, x\right): x^{0}=u(x), x \in B_{\delta}\left(x_{0}\right)\right\}
$$

lies in the past of the slice $M\left(d_{0}+s\right)$ in view of $(7.45)$, hence if we write

$$
\hat{M}(s)=\operatorname{graph} u
$$

in $B_{\delta}\left(x_{0}\right)$, then

$$
u \leq u\left(x_{0}\right)=d_{0}+s .
$$

The hypersurface $\hat{M}(s)$ therefore touches the hypersurface $M\left(d_{0}+s\right)$ from below. Since

$$
\bar{H}\left(d_{0}+s\right)=H_{\left.\right|_{M\left(d_{0}+s\right)}} \geq c_{0}
$$

and

$$
H_{\left.\right|_{\hat{M}(s)}} \leq c_{0}
$$

we deduce

$$
\bar{H}\left(d_{0}+s\right)-H_{\left.\right|_{\hat{M}(s)}} \geq 0
$$

in $B_{\delta}\left(x_{0}\right)$. The left-hand side can be written as a uniformly elliptic equation for the non-negative function

$$
\tilde{u}=d_{0}+s-u \geq 0
$$

such that

$$
-D_{i}\left(a^{i j} D_{j} \tilde{u}\right)+b^{i} \tilde{u}_{i}+c \tilde{u} \geq 0
$$

in $\mathrm{B}_{\delta}\left(x_{0}\right)$, where we used (6.19) on page 33 , (5.39) on page $25,(6.42)$ on page 35 and the identity

$$
\tilde{v}-1=\frac{\tilde{v}^{2}}{\tilde{v}+1}|D u|^{2} .
$$

The weak Harnack inequality then implies

$$
u(x)=d_{0}+s \quad \forall x \in B_{\delta}\left(x_{0}\right) .
$$

But then the tubular neighbourhood contains the full cylinder

$$
\left[0, d_{0}\right] \times B_{\delta}\left(x_{0}\right)
$$

and for every point

$$
p=(0, x) \in M_{2}, \quad x \in B_{\delta}\left(x_{0}\right),
$$

there exists a corresponding point $q \in M_{1}$ such that

$$
d_{0}=d\left(M_{2}, M_{1}\right)=d(p, q)
$$


Now we can derive a contradiction. Let $\Lambda$ be defined by

$$
\Lambda=\left\{p \in M_{2}: \exists q \in M_{1} \text { with } d(p, q)=d_{0}\right\} .
$$

Obviously, $\Lambda \neq \emptyset$ and closed and we have just proved that $\Lambda$ is also open, hence

$$
\Lambda=M_{2}
$$

and any future directed orthogonal geodesic emanating from $M_{2}$ will meet $M_{1}$ and realize the distance $d_{0}$, which certainly contradicts the estimate (7.24).

Corollary 7.3. The functions $u(t, \cdot)$ the graphs of which have constant mean curvature $\hat{H}(t)$ and which tend to $t$ at spatial infinity are continuous in $t$.

Proof. Obvious.

Thus, there exists a family

$$
M_{t}=\operatorname{graph} u(t, \cdot), \quad t \in I,
$$

of constant mean curvature hypersurfaces which are monotonically ordered by (7.13). We shall now prove that they form a foliation of $N$.

Theorem 7.4. The hypersurfaces $M_{t}, t \in I$, provide a foliation of $N$.

Proof. We argue by contradiction and assume that there exists a point $p_{0}=$ $\left(t_{0}, x_{0}\right)$ such that

$$
p_{0} \notin \bigcup_{t \in I} M_{t}
$$

Let $\Lambda_{ \pm}$be defined by

$$
\Lambda_{+}=\left\{t \in I: u\left(t, x_{0}\right)>t_{0}\right\}
$$

and

$$
\Lambda_{-}=\left\{t \in I: u\left(t, x_{0}\right)<t_{0}\right) .
$$

Both sets are nonempty because of Lemma 7.1.

Next, let $u_{2}$ be defined by

$$
u_{2}=\inf _{t \in \Lambda_{+}} u(t, \cdot)
$$

and

$$
u_{1}=\sup _{t \in \Lambda_{-}} u(t, \cdot)
$$

then

$$
\lim _{r(x) \rightarrow \infty} u_{2}(x)=t_{2}=\inf _{t \in \Lambda_{+}} t
$$


and

$$
\lim _{r(x) \rightarrow \infty} u_{1}(x)=t_{1}=\sup _{t \in \Lambda_{-}} t
$$

in view of (7.24) and the respective mean curvatures of

$$
M_{i}=\operatorname{graph} u_{i}, \quad i=1,2,
$$

are $\hat{H}\left(t_{i}\right)$. Moreover, because of the monotonicity

$$
\begin{aligned}
& t_{1} \leq t_{2}, \\
& u_{1} \leq u_{2}
\end{aligned}
$$

and

$$
u_{1}\left(x_{0}\right) \leq t_{0} \leq u_{2}\left(x_{0}\right) .
$$

Because of Lemma 7.2 we know

$$
u_{i}=u\left(t_{i}, \cdot\right)
$$

Thus, the assumption

$$
t_{1}<t_{2}
$$

would lead to a contradiction by simply picking any $\bar{t}$

$$
t_{1}<\bar{t}<t_{2} \text {. }
$$

The existence of graph $u(\bar{t}, \cdot)$ would then contradict the definition of $u_{1}$ or $u_{2}$. Hence, we conclude

$$
t_{1}=t_{2}
$$

and consequently

$$
u_{1}=u_{2}
$$

which implies

$$
u_{1}\left(t_{0}\right)=t_{0},
$$

i.e., the theorem is proved.

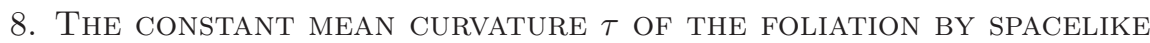
HYPERSURFACES IS A SMOOTH GLOBAL TIME FUNCTION

After having proved the existence of a unique foliation by spacelike CMC hypersurfaces $M_{t}, t \in I$, we want to show that the mean curvature

$$
\tau=\hat{H}(t)
$$

of the foliation hypersurfaces is a smooth global time function, provided the spacetime $N$ is smooth. First, $\tau$ is certainly a continuous time function, since the level hypersurfaces

$$
\{\tau=\text { const }\}
$$


are $C^{3, \alpha}$ spacelike hypersurfaces, or even smooth hypersurfaces, if $N$ is smooth, and $\tau$ is also continuous, since

$$
\tau\left(x^{0}, x\right)=H_{\left.\right|_{M(t)}} \quad \forall\left(x^{0}, x\right) \in N,
$$

where

$$
\left(x^{0}, x\right) \in M_{t}
$$

or, equivalently,

$$
x^{0}=u(t, x),
$$

and we know that, if

$$
\left(x_{k}^{0}, x_{k}\right) \rightarrow\left(x_{0}^{0}, x_{0}\right) \quad \text { in } N
$$

such that

$$
\left(x_{k}^{0}, x_{k}\right) \in M_{t_{k}},
$$

then

$$
t_{k} \rightarrow t_{0}
$$

and

$$
\left(x_{0}^{0}, x_{0}\right) \in M_{t_{0}},
$$

or equivalently,

$$
x_{0}^{0}=u\left(t_{0}, x_{0}\right),
$$

in view of Corollary 7.3 on page 44 .

In order to prove that $\tau$ is of class $C^{1}$, it suffices to show

$$
u(\cdot, x) \in C^{1}(I) \quad \forall x \in \mathcal{S}_{0}
$$

and

$$
\dot{u}=\frac{\partial u}{\partial t}>0 .
$$

Here, we already used the fact that the function in (8.11) is strictly monotone growing in $t$.

Indeed, if (8.1) and (8.12) are valid, then we deduce from (8.5)

$$
\frac{\partial x^{0}}{\partial t}=\dot{u}>0 .
$$

On the other hand, the function $\hat{H}$ in (8.1) is a diffeomorphism, i.e.,

$$
\frac{\partial \tau}{\partial t}=\hat{H}^{\prime}>0
$$

and hence we conclude

$$
\frac{\partial x^{0}}{\partial \tau}=\frac{\partial x^{0}}{\partial t} \frac{\partial t}{\partial \tau}=\dot{u}\left(\hat{H}^{\prime}\right)^{-1}>0
$$

and

$$
\frac{\partial \tau}{\partial x^{0}}=\frac{\hat{H}^{\prime}}{\dot{u}}
$$


Once we have proved (8.11) and (8.12) the conclusion $\tau$ is smooth if $N$ is smooth follows immediately as we shall show.

Thus, let us prove (8.11) and (8.12) by showing that these relations are valid for the solutions $u=u_{R}$ of the Dirichlet problems (3.1) on page 9. Since we shall also derive

$$
0<\dot{u}_{R}(t, x) \leq c \quad \forall x \in B_{R},
$$

where the constant $c$ is dependent of $R$ and also independent of $t$, als long as $t$ stays in a compact subset of $I$, we then conclude

$$
0 \leq \dot{u} \leq c \quad \forall x \in \mathcal{S}_{0},
$$

and the weak Harnack inequality will then allow us to actually deduce

$$
0<\dot{u} \text {. }
$$

Thus, let $u=u_{R}(t, x)$ be a solution of (3.1) with boundary values $t$ and mean curvature $\hat{H}$. First, we need to prove the uniqueness of $u$.

Lemma 8.1. The solution $u \in C^{3, \alpha}\left(\bar{B}_{R}\right)$ of the Dirichlet problem (3.1) is unique.

Proof. Let $M_{i}=\operatorname{graph} u_{i}, i=1,2$, be two solutions of (3.1) and assume that there exists $x \in B_{R}$ such that

$$
u_{2}(x)<u_{1}(x) .
$$

Then, there exists a maximizing future directed timelike geodesic $\gamma=\left(\gamma^{\alpha}\right)$ from $\bar{M}_{2}$ to $\bar{M}_{1}$ and $p_{i} \in \bar{M}_{i}$ such that

$$
0<d_{0}=d\left(M_{2}, M_{1}\right)=d\left(p_{2}, p_{1}\right),
$$

where

$$
p_{i}=u_{i}\left(x_{i}\right), \quad x_{i} \in \bar{B}_{R}
$$

Note that

$$
d_{0}=\sup \left\{d\left(x_{2}, x_{1}\right): x_{2} \in M_{2}, x_{1} \in M_{1}\right\}
$$

and

$$
0<d\left(p_{2}, p_{1}\right) \leq d_{0}
$$

where

$$
p_{i}=\left(u_{i}(x), x\right), \quad i=1,2 .
$$

Since the $\bar{M}_{i}$ are compact and $d$ continuous, the supremum is achieved, hence (8.21) is valid and, furthermore, there exists a future directed timelike geodesic from $p_{2}$ to $p_{1}$, cf. [10, Proposition 6.7.1, p. 212].

Let $Q$ be the open cylinder

$$
Q=I \times B_{R}
$$

and let $\gamma$ be parametrized in the interval $[0,1]$. Suppose

$$
p_{1} \in \partial Q,
$$


then

$$
p_{2} \notin \partial Q,
$$

since $\gamma$ is future directed and hence

$$
\gamma^{0}(\tau)<\gamma^{0}(1)=t \quad \forall 0 \leq \tau<1 .
$$

Let $\tau_{0}$ be the first

$$
\tau \in(0,1]
$$

such that

$$
\gamma(\tau) \in \partial Q
$$

If $\tau_{0}=1$, then

$$
\gamma(\tau) \in \bar{Q} \quad \forall \tau \in[0,1] .
$$

Since $M_{2}$ can be extended as a spacelike graph $\tilde{M}_{2}$ over a slightly larger ball $\bar{B}_{R+\epsilon} \gamma$ would then be a future directed curve completely contained in the open cylinder

$$
\tilde{Q}=I \times B_{R+\epsilon}
$$

with endpoints $p_{i} \in \tilde{M}_{2}$, which leads to a contradiction, in view of Lemma 3.2 on page 11 .

Thus, let us assume

$$
\tau_{0}<1,
$$

then

$$
\gamma^{0}\left(\tau_{0}\right)<u_{2}\left(\gamma^{i}\left(\tau_{0}\right)\right)=t
$$

and we can define a new future directed broken curve $\tilde{\gamma}$ completely contained in $\tilde{Q}$ with endpoints in $\tilde{M}_{2}$, cf. part (ii) of the proof of Lemma 3.1 on page 9 , which again leads to a contradiction as before.

By switching the time orientation the previous arguments also exclude the case

$$
p_{2} \in \partial Q .
$$

Thus, we may assume

$$
p_{i} \in M_{i}, \quad i=1,2 .
$$

But then we are in the same situation as in the proof of Lemma 7.2 after equation (7.39) on page 41 and the arguments there lead to a contradiction.

Corollary 8.2. Let us write solutions $u_{R}$ of (3.1) in the form

$$
u_{R}=u_{R}(t, x), \quad(t, x) \in I \times \bar{B}_{R},
$$

then $u_{R}$ is continuous in $t$.

Proof. Obvious. 
Next, let us prove:

Lemma 8.3. The function $u_{R}(\cdot, x)$ belongs to $C^{1}(I)$ for all $x \in \bar{B}_{R}$.

Proof. Let $t_{0} \in I$ be arbitrary, define

$$
M_{t}=\operatorname{graph} u_{R}(t, \cdot)
$$

and set

$$
M_{0}=M_{t_{0}} .
$$

The $\bar{M}_{t}$ are of class $C^{3, \alpha}$. Let $\mathcal{U}$ be a tubular neighbourhood of $M_{0}$ and $\vartheta$ the signed distance function to $M_{0}$ such that $\vartheta$ is a time function in $\mathcal{U}$; $\vartheta$ is also the time coordinate of a normal Gaussian coordinate system $\left(\vartheta, x^{i}\right)$ in $\mathcal{U}$, cf. [6, Theorem 12.5.13]. Since $M_{0}$ is a graph over $B_{R}$ we may consider the $\left(x^{i}\right)$ to be local coordinates in $B_{R}$. Note that

$$
\vartheta \in C^{3, \alpha}(\mathcal{U}) \text {. }
$$

Since we can extend $M_{0}$ to be a graph over a slightly larger ball we shall assume that $\mathcal{U}$ covers $\bar{M}_{0}$. Moreover, we know that there is $\delta>0$ such that

$$
\bar{M}_{t} \subset \mathcal{U} \quad \forall t \in\left(t_{0}-\delta, t_{0}+\delta\right) .
$$

In terms of $\left(x^{0}, x\right) \vartheta$ can be expressed as

$$
\vartheta=\tilde{\varphi}\left(x^{0}, x\right), \quad \tilde{\varphi} \in C^{3, \alpha},
$$

and similarly

$$
x^{0}=\varphi(\vartheta, x), \quad \varphi \in C^{3, \alpha} .
$$

Note that the spatial coordinates are the same in both coordinate systems. The slice

$$
\left\{x^{0}=t\right\} \cap \mathcal{U}
$$

can then be expressed as the graph

$$
\{(\vartheta, x): \vartheta=\tilde{\varphi}(t, x)\}
$$

and the hypersurfaces $M_{t}$ in (8.42) as

$$
\{(\vartheta, x): \vartheta=\tilde{\varphi}(u(t, x), x) \equiv \tilde{u}(t, x)\},
$$

and similarly

$$
u(t, x)=\varphi(\tilde{u}(t, x), x) .
$$

We shall now prove that

$$
\tilde{u}(\cdot, x) \in C^{1}\left(I_{\delta_{0}}\right),
$$

where

$$
I_{\delta_{0}}=\left(t_{0}-\delta_{0}, t_{0}+\delta_{0}\right)
$$

for some small

$$
0<\delta_{0}<\delta
$$


Let us define the function space

$$
C_{0}^{3, \alpha}\left(\bar{B}_{R}\right)=\left\{u \in C^{3, \alpha}\left(\bar{B}_{R}\right): u_{\left.\right|_{\partial B_{R}}}=0\right\} .
$$

In view of (8.45) and (8.46) the graph

$$
\{\vartheta=\tilde{\varphi}(t, x)\}
$$

represents the graph

$$
\left\{x^{0}=t\right\}
$$

in the new coordinate, hence

$$
0=\tilde{\varphi}\left(t_{0}, x\right) \quad \forall x \in \partial B_{R}
$$

and by continuity

$$
-\epsilon_{1}<\tilde{\varphi}(t, x)<\epsilon_{1} \quad \forall(t, x) \in \mathscr{U}_{\delta_{1}},
$$

where

$$
\mathscr{U}_{\delta_{1}}=\left(t_{0}-\delta_{1}, t_{0}+\delta_{1}\right) \times\left\{x \in \bar{B}_{R}: R-\rho(x)<\delta_{1}\right\} .
$$

Let

$$
\chi \in C_{c}^{\infty}\left(-\delta_{1}, \delta_{1}\right)
$$

be a real cut-off function such that

$$
\chi(0)=1 \wedge 0 \leq \chi \leq 1
$$

and set

$$
w=\chi(R-\rho(x)),
$$

then

$$
w(x) \tilde{\varphi}(\cdot, x) \in C^{1}\left(I_{\delta_{1}}\right) \quad \forall x \in \bar{B},
$$

where

$$
I_{\delta_{1}}=\left(t_{0}-\delta_{1}, t_{0}+\delta_{1}\right),
$$

and

$$
R(w \tilde{\varphi}) \subset J_{\delta}=(-\delta, \delta) \quad \forall(t, x) \in I_{\delta_{1}} \times \bar{B}_{R} .
$$

Hence, the last relation is also valid for all

$$
\tilde{u}+w \tilde{\varphi}(t, \cdot) \quad \forall t \in I_{\delta_{1}},
$$

where

$$
\tilde{u} \in \mathscr{B}_{\epsilon_{0}}(-w \tilde{\varphi}) \subset C_{0}^{3, \alpha}\left(\bar{B}_{R}\right),
$$

if $\epsilon_{0}$ and $\delta_{1}$ are small enough, and

$$
M=\operatorname{graph}(\tilde{u}+w \tilde{\varphi}(t, \cdot))
$$

is a spacelike graph in $(\vartheta, x)$ which is contained in the tubular neighbourhood $\mathcal{U}$ which can be expressed in the new coordinate system as a cylinder

$$
\mathcal{U}=(-\delta, \delta) \times B_{R+\epsilon} .
$$


If the hypersurface $M$ is viewed as a graph in $\left(x^{0}, x\right)$,

$$
M=\operatorname{graph} u,
$$

then

$$
u_{\left.\right|_{\partial B_{R}}}=t .
$$

Indeed, according to (8.48)

$$
u(x)=\varphi(\tilde{u}(x)+w \tilde{\varphi}(t, x), x)
$$

and

$$
u(x)=\varphi(\tilde{\varphi}(t, x), x)=t \quad \forall x \in \partial B_{R} .
$$

We have

$$
M_{0}=\left\{(\vartheta, x): \vartheta=0, x \in B_{R}\right\}
$$

i.e.,

$$
M_{0}=\operatorname{graph}\left(-w \tilde{\varphi}\left(t_{0}, \cdot\right)+w \tilde{\varphi}\left(t_{0}, \cdot\right)\right) .
$$

Hence, for all $\tilde{u}$ in (8.65) the hypersurfaces

$$
M(t, \tilde{u})=\operatorname{graph}(\tilde{u}+w \tilde{\varphi}(t, \cdot)), \quad t \in I_{\delta_{1}},
$$

are spacelike and contained in $\mathcal{U}$. Thus, we can define the operator

$$
\begin{gathered}
G: I_{\delta_{1}} \times \mathscr{B}_{\epsilon_{0}}\left(-w \varphi\left(t_{0}, \cdot\right)\right) \rightarrow C^{1, \alpha}\left(\bar{B}_{r}\right), \\
G(t, \tilde{u})=H_{\left.\right|_{M(t, \tilde{u})}}-\hat{H}(t)
\end{gathered}
$$

and consider the equation

$$
G(t, \tilde{u})=0
$$

which describes the Dirichlet problem (3.1) on page 9 in the coordinate system $(\vartheta, x)$ as an implicit function equation. We know that $G$ is at least of class $C^{1}$ in $(t, \tilde{u})$ and that

$$
G\left(t_{0},-w \tilde{\varphi}\left(t_{0}, \cdot\right)\right)=0
$$

and

$$
D_{2} G\left(t_{0},-w \tilde{\varphi}\left(t_{0}, \cdot\right)\right)=L,
$$

where $L$,

$$
L: C_{0}^{3, \alpha}\left(\bar{B}_{R}\right) \rightarrow C^{1, \alpha}\left(\bar{B}_{R}\right),
$$

is the elliptic differential operator

$$
L \tilde{u}=-\Delta \tilde{u}+\left(|A|^{2}+\bar{R}_{\alpha \beta} \nu^{\alpha} \nu^{\beta}\right) \tilde{u} .
$$

Here, the Laplacian is defined by the metric $g_{i j}$ in $M_{0}$,

$$
|A|^{2}=h^{i j} h_{i j},
$$

and $\left(\nu^{\alpha}\right)$ is the normal of $M_{0}$.

Since

$$
N(L)=\{0\}
$$


$L$ is a topological homeomorphism, and hence, we conclude from the implicit function theorem that there exists a small neighbourhood

$$
I_{\epsilon}\left(t_{0}\right) \subset I_{\delta_{1}}
$$

and a uniquely determined function

$$
\theta \in C^{1}\left(I_{\epsilon}\left(t_{0}\right), C_{0}^{3, \alpha}\left(\bar{B}_{R}\right)\right)
$$

such that

$$
G(t, \theta(t))=0 \quad \forall \in I_{\epsilon}\left(t_{0}\right) .
$$

Writing

$$
\tilde{u}(t, x)=\theta(t)+w \tilde{\varphi}(t, \cdot)
$$

we conclude that $\tilde{u}(\cdot, x)$ is of class $C^{1}$ in the interval $I_{\epsilon}\left(t_{0}\right)$. Expressing the graphs in the original coordinates we obtain, in view of (8.48),

$$
u(\cdot, x)=\varphi(\tilde{u}(\cdot, x), x)
$$

is of class $C^{1}$ in the intervall $I_{\epsilon}\left(t_{0}\right)$.

Corollary 8.4. From (8.87) we conclude

$$
\dot{u}\left(t_{0}, x\right)=\dot{\varphi}(0, x) \dot{\tilde{u}}\left(t_{0}, x\right)=e^{-\psi} \tilde{v} \dot{\tilde{u}}\left(t_{0}, x\right) \quad \forall x \in \bar{B}_{R} .
$$

Proof. We only have to prove that

$$
\dot{\varphi}(0, x)=e^{-\psi} \tilde{v} .
$$

Let us recall that the tubular neighbourhood is defined by the geodesic flow $\gamma(\vartheta, x)=\left(\gamma^{\alpha}\right)$ defined in the coordinates $\left(x^{0}, x^{i}\right)$. Here $\vartheta$ is the signed arc length which is identical to the signed distance function of $M_{0}$, the initial values of the flow at $\vartheta=0$ are

$$
\gamma(0, x)=\left(u\left(t_{0}, x\right), x\right) \wedge \dot{\gamma}(0, x)=-\nu(x),
$$

where $\nu$ is the past directed normal of $M_{0}$. Thus, the flow is future directed. The function $\varphi(\vartheta, x)$ in the relation (8.44) is then identical to

$$
\varphi(\vartheta, x)=\gamma^{0}(\vartheta, x)
$$

hence

$$
\dot{\varphi}(0, x)=\dot{\gamma}^{0}(0, x)=-\nu^{0}=e^{-\psi} \tilde{v} .
$$

Proposition 8.5. Let $\tilde{u}(t, x)=\tilde{u}_{R}(t, x)$ be a solution of the Dirichlet problem (3.1) in the tubular neighbourhood $\mathcal{U}$ of

$$
M_{0}=\operatorname{graph} u_{R}\left(t_{0}, \cdot\right),
$$

then

$$
0 \leq \dot{\tilde{u}}\left(t_{0}, x\right) \leq c \quad \forall x \in B_{R},
$$

where $c$ depends on known estimates but not on $R$. 
Proof. Let us differentiate the equations (8.85) and (8.86) with respect to $t$ and evaluate at $t=t_{0}$. Then we obtain

$$
-\Delta \dot{\tilde{u}}\left(t_{0}\right)+\left(|A|^{2}+\bar{R}_{\alpha \beta} \nu^{\alpha} \nu^{\beta}\right) \dot{\tilde{u}}\left(t_{0}\right)=\hat{H}^{\prime}\left(t_{0}\right)
$$

as well as the boundary condition

$$
\dot{\tilde{u}}\left(t_{0}, x\right)_{\left.\right|_{\partial B_{R}}}=\dot{\tilde{\varphi}}\left(t_{0}, x\right)_{\left.\right|_{\partial B_{R}}},
$$

where

$$
\hat{H}^{\prime}=\frac{\partial \hat{H}}{\partial t}>0
$$

Let us first note that

$$
t=\varphi(\tilde{\varphi}(t, x), x)
$$

and therefore

$$
1=\dot{\varphi}(0, x) \dot{\tilde{\varphi}}\left(t_{0}, x\right)
$$

hence,

$$
\dot{\tilde{\varphi}}(0, x)=e^{\psi} v
$$

in view of (8.92), i.e., the boundary values of $\dot{\tilde{u}}\left(t_{0}, x\right)$ are uniformly bounded and tend to 1 if $R$ tends to infinity.

Secondly, the coefficient in (8.95) is non-negative

$$
|A|^{2}+\bar{R}_{\alpha \beta} \nu^{\alpha} \nu^{\beta} \geq 0
$$

and strictly positive for large $r(x)$, or equivalently, $\rho(x)$,

$$
|A|^{2}+\bar{R}_{\alpha \beta} \nu^{\alpha} \nu^{\beta} \geq c_{0}>0 \quad \forall x \in\left\{\rho(x) \geq R_{1}\right\},
$$

where $R_{1}$ is sufficiently large, cf. Corollary 6.4 on page 38 .

Let us now estimate $\dot{\tilde{u}}=\dot{\tilde{u}}\left(t_{0}, \cdot\right)$ from above. Assuming $R>R_{1}$ we choose $k_{0} \in \mathbb{R}$ such that

$$
k_{0} \geq \sup _{\partial B_{R}} e^{\psi} v
$$

and define for $k \geq k_{0}$

$$
\eta=\max (\dot{\tilde{u}}-k, 0) \in H_{0}^{1,2}\left(B_{R}\right) .
$$

Multiplying (8.95) by $\eta$ and integrating by parts we obtain

$$
\int_{B_{R}}|D \eta|^{2}+\int_{B_{R}}\left(|A|^{2}+\bar{R}_{\alpha \beta} \nu^{\alpha} \nu^{\beta}\right) \dot{\tilde{u}} \eta=\int_{B_{R}} \hat{H}^{\prime} \eta .
$$

Choosing at the moment $k=k_{0}$ and assuming furthermore that $k_{0}$ satisfies besides (8.103) also

$$
c_{0} k_{0} \geq \hat{H}^{\prime}\left(t_{0}\right)+1
$$

we infer

$$
\int_{B_{R}}|D \eta|^{2}+\int_{B_{R} \backslash B_{R_{1}}} \eta \leq \int_{B_{R_{1}}} \hat{H}^{\prime} \eta
$$


The right-hand side can be estimated by

$$
\int_{B_{R_{1}}} \hat{H}^{\prime} \eta \leq \hat{H}^{\prime}\left|B_{R_{1}}\right|^{\frac{2 n}{n+2}}\left(\int_{B_{R}}|\eta|^{\frac{2 n}{n-2}}\right)^{\frac{n-2}{2 n}} .
$$

On the other hand, in view of the Sobolev embedding theorem, we have

$$
\left(\int_{B_{R}}|\eta|^{\frac{2 n}{n-2}}\right)^{\frac{n-2}{n}} \leq c \int_{B_{R}}|D \eta|^{2}
$$

and thus we conclude

$$
\int_{B_{R}}|D \eta|^{2} \leq c=\text { const }
$$

independent of $R$. This inequality is also valid if $\eta$ is defined as in (8.104) for $k \geq k_{0}$.

Consider now $k \geq k_{0}$ to be arbitrary and let us look again at the inequality (8.107). Define

$$
A(k)=\left\{x \in B_{R}: \dot{\tilde{u}}>k\right\}
$$

then we deduce

$$
\left(\int_{B_{R}}|\eta|^{\frac{2 n}{n-2}}\right)^{\frac{n-2}{n}} \leq c|A(k)|^{\frac{n+2}{2 n}}\left(\int_{B_{R}}|\eta|^{\frac{2 n}{n-2}}\right)^{\frac{n-2}{2 n}}
$$

implying

$$
\left(\int_{B_{R}}|\eta|^{\frac{2 n}{n-2}}\right)^{\frac{n-2}{2 n}} \leq c|A(k)|^{\frac{n+2}{2 n}} .
$$

Next, let $h>k$ then

$$
(h-k)|A(h)| \leq \int_{B_{R}} \eta \leq c|A(k)|^{1+\frac{2}{n}} \quad \forall h>k \geq k_{0}
$$

from which we conclude, in view of a lemma due to Stampacchia, cf. [13, Lemma 4.1, p. 93],

$$
\dot{\tilde{u}} \leq k_{0}+d
$$

where

$$
d=c\left|A\left(k_{0}\right)\right|^{\frac{2}{n}} 2^{\frac{n}{2}+1} .
$$

To prove the uniform boundedness of $\left|A\left(k_{0}\right)\right|$ we assume that $k_{0}$ is so large that

$$
k_{1}=\frac{k_{0}}{2}-1
$$

also satisfies the inequalities in (8.103) and (8.106) if we replace $k_{0}$ by $k_{1}$. Then

$$
\int_{B_{R}} \eta_{0}^{\frac{2 n}{n-2}} \leq c
$$


where

$$
\eta_{0}=\max \left(\dot{\tilde{u}}-k_{1}, 0\right)
$$

and we deduce

$$
\begin{aligned}
\left|A\left(k_{0}\right)\right| & \leq \frac{1}{\left(\frac{k_{0}}{2}+1\right)^{\frac{2 n}{n-2}}} \int_{A\left(k_{0}\right)}\left(u-k_{1}\right)^{\frac{2 n}{n-2}} \\
& \leq \frac{1}{\left(\frac{k_{0}}{2}+1\right)^{\frac{2 n}{n-2}}} \int_{B_{R}}\left|\eta_{0}\right|^{\frac{2 n}{n-2}} .
\end{aligned}
$$

Proposition 8.6. Let $\dot{\tilde{u}}\left(t_{0}, x\right)$ be the solution of (8.95) and the corresponding boundary condition, then

$$
\left|\dot{\tilde{u}}\left(t_{0}, \cdot\right)\right|_{2, \alpha, \bar{B}_{R}} \leq c
$$

where $c$ only depends on already proven estimates but not on $R$.

Proof. The proof is identical to the proof of Lemma 6.2 on page 35 .

If we want to improve the regularity of $\tilde{u}(t, x)$ we have to assume a higher regularity of $N$. At the moment we only assume $N$ to be of class $C^{3, \alpha}$. Thus, let us suppose $N$ to be of class $C^{m, \alpha}, m \geq 3,0<\alpha<1$. Then, the original coordinates $\left(x^{\alpha}\right)$ with the time function $x^{0}$ are of class $C^{m, \alpha}$, the metric $\bar{g}_{\alpha \beta}$ of class $C^{m-1, \alpha}$ and the second fundamental form $\bar{h}_{i j}$ of the coordinate slices and the Riemann curvature tensor are of class $C^{m-2, \alpha}$ with uniform bounds.

The solutions $u(t, x)=u_{R}(t, x)$ of the Dirichlet problems (3.1) on page 9 are then of class $C^{m, \alpha}\left(\bar{B}_{R}\right)$ with uniform bounds independent of $R$. The estimates, of course, depend on $m$.

First, let us prove:

Lemma 8.7. Let $N$ be of class $C^{m, \alpha}, m \geq 3,0<\alpha<1$, with uniform bounds, $u(t, x)=u_{R}(t, x)$ a solution of the Dirichlet problem (3.1) with boundary value $t_{0}$ and let

$$
M_{0}=\operatorname{graph} u\left(t_{0}, \cdot\right) .
$$

Let $(\vartheta, x)$ be the normal Gaussian coordinate system corresponding to a tubular neighbourhood $\mathcal{U}$ of $\bar{M}_{0}$, then $\vartheta$ and the transformation maps

$$
x^{0}=\varphi(\vartheta, x)
$$

and its inverse

$$
\vartheta=\tilde{\varphi}\left(x^{0}, x\right)
$$

are of class $C^{m, \alpha}$ with respect to the indicated variables such that the corresponding $C^{m, \alpha}$-norms, evaluated at $\bar{M}_{0}$, are uniformly bounded independent of $R$. 
Proof. It suffices to prove the claim for $\varphi$. As we mention before, the new coordinate system is created with the help of the geodesic flow $\gamma(\vartheta, x)$ with initial hypersurface $\bar{M}_{0}$ and initial values

$$
\gamma(0, x)=(u(x), x) \wedge \dot{\gamma}(0, x)=-\nu(x) .
$$

$\vartheta$ is the signed arc length, which will be negative in the past of $M_{0}, \nu$ is supposed to be the past directed normal of $M_{0}$. The map $\varphi$ is defined by

$$
x^{0}=\gamma^{0}(\vartheta, x) \equiv \varphi(\vartheta, x) .
$$

In view of (8.125) we immediately obtain

$$
\gamma(0, \cdot) \in C^{m, \alpha}\left(\bar{B}_{R}\right) \wedge \dot{\gamma}(0, \cdot) \in C^{m-1, \alpha}\left(\bar{B}_{R}\right)
$$

with uniform bounds independent of $R$ and from the geodesic equation and the assumption on $N$ we recursively deduce

$$
D_{\vartheta}^{k} \gamma(0, \cdot) \in C^{m-k, \alpha}\left(\bar{B}_{R}\right) \quad \forall 0 \leq k \leq m,
$$

again with uniform bounds independent of $R$.

We can now prove:

Theorem 8.8. Let $N$ be of class $C^{m, \alpha}, m \geq 3,0<\alpha<1$, and let $\tilde{u}(t, x)=\tilde{u}_{R}(t, x)$ be the solutions of the Dirichlet problems (3.1) expressed in the new coordinates $(\vartheta, x)$. Then $\tilde{u}$ is of class $C^{m-2}$ with respect to $t$ and

$$
\frac{\partial^{k} \tilde{u}}{(\partial t)^{k}}\left(t_{0}, \cdot\right) \in C^{m-k, \alpha}\left(\bar{B}_{R}\right) \quad \forall 0 \leq k \leq m-2
$$

with uniform bounds independent of $R$.

Proof. We shall prove the theorem inductively; for $k=0,1$ this has already been proved before, cf. Proposition 8.6 and apply the Schauder estimates to the solution of (8.95) with boundary values given in (8.96).

To prove the claim for $k=2$, let us consider the elliptic differential equation satisfied by $\tilde{u}(t, \cdot)$ for $t$ near $t_{0}$

$$
\begin{aligned}
\hat{H}(t) \tilde{v} & =-g^{i j} \tilde{u}_{i j}+g^{i j} \bar{h}_{i j} \\
& =-g^{i j} \tilde{u}_{i j}+\bar{H}+\tilde{v}^{2} \bar{h}_{i j} \check{\tilde{u}}^{i} \check{\tilde{u}}^{j} .
\end{aligned}
$$

Here, $g_{i j}$ is the induced metric of $M_{t}=\operatorname{graph} \tilde{u}(t, \cdot)$ and the ambient metric is expressed in normal Gaussian coordinates

$$
d \bar{s}^{2}=-d \vartheta^{2}+\sigma_{i j}(\vartheta, x) d x^{i} d x^{j}
$$

in the tubular neighbourhood $\mathcal{U}$ of $M_{0}$. At $t=t_{0}$ we know

$$
\tilde{u}\left(t_{0}, \cdot\right) \equiv 0 \text {. }
$$

Differentiating (8.130) twice with respect to $t$, the differentiability is due to the implicit function theorem, and evaluating the result at $t=t_{0}$ we obtain

$$
\hat{H}^{\prime \prime}+F=-g^{i j} \ddot{\tilde{u}}_{i j}+\dot{\bar{H}} \ddot{\tilde{u}}
$$


where

$$
F=F\left(x, \tilde{u}, \dot{\tilde{u}}, D \tilde{u}, D \dot{\tilde{u}}, D^{2} \tilde{u}, D^{2} \dot{\tilde{u}}\right) \in C^{m-3, \alpha}\left(\bar{B}_{R}\right)
$$

with uniform bounds independent of $R$. The proof of Proposition 8.5 then yields

$$
\left|\ddot{\tilde{u}}\left(t_{0}, \cdot\right)\right| \leq c \quad \text { in } \bar{B}_{R},
$$

where, now, we also have to estimate $\ddot{\tilde{u}}$ from below. Combining this estimate with the Schauder estimates we conclude

$$
\left|\ddot{\tilde{u}}\left(t_{0}, \cdot\right)\right|_{m-2, \alpha, \bar{B}_{R}} \leq c,
$$

since

$$
\ddot{\tilde{u}}_{\left.\right|_{\partial B_{R}}} \in C^{m-2, \alpha} .
$$
Set

The same arguments also apply when higher derivatives are considered.

$$
\tilde{u}^{(k)}=\frac{\partial^{k} \tilde{u}}{(\partial t)^{k}}\left(t_{0}, \cdot\right)
$$

then

$$
D_{t}^{k} \hat{H}+F=-\Delta \tilde{u}^{(k)}+\dot{\bar{H}} \tilde{u}^{(k)},
$$

where, now, $F$ depends on

$$
F=F\left(x, \tilde{u}, \dot{\tilde{u}}, \ldots, D^{2} \tilde{u}^{(k-1)}\right) \in C^{m-k, \alpha}\left(\bar{B}_{R}\right),
$$

and the boundary values are of class $C^{m-k, \alpha}$.

Corollary 8.9. The results of Theorem 8.8 are also valid for $u_{R}\left(t_{0}, x\right)$, in view of Lemma 8.7, see also Corollary 8.4. Moreover, since $t_{0}$ is arbitrary, these estimates are valid for any $t$ and they are uniform provided $t$ ranges in a compact subset of $I$.

Letting $R$ tend to infinity we then deduce:

Theorem 8.10. The functions

$$
u(t, x), \quad(t, x) \in I \times \mathcal{S}_{0},
$$

describing the foliation hypersurfaces $M_{t}, t \in I$, are of class $C^{m-3,1}$ in $t$ such that

$$
D_{t}^{k} u \in C^{m-k, \alpha}\left(\mathcal{S}_{0}\right) \quad \forall 0 \leq k \leq m-3,
$$

if $N$ is of class $C^{m, \alpha}, m \geq 3,0<\alpha<1$; if $N$ is smooth, i.e., $m=\infty$, then $u$ is also smooth in the variables $(t, x)$ and the mean curvature function $\tau=\tau\left(x^{0}, x\right)$ is a smooth time function.

Proof. This immediately follows from Lemma 6.2 on page 35 and the considerations at the beginning of this section. 


\section{Appendix: LipsChitz CONTINUOUs SOlutions ARE REGUlAR}

In this appendix we want to prove that Lipschitz continuous solutions of the Dirichlet problems (3.1) on page 9 or of the related equation (5.96) on page 30 are of class $H^{2, p}$ or even more regular depending on the data.

We shall use the assumptions stated at the beginning of the proof of Lemma 5.4 and consider an allowed Lipschitz continuous solution $u$ of (5.96) in $B_{R}$ with vanishing boundary values which satisfies

$$
|D u|^{2}=\sigma^{i j}(u, x) D_{i} u D_{j} u \leq 1-\delta^{2},
$$

and

$$
m_{1} \leq u \leq m_{2}, \quad m_{i} \in I .
$$

The equation (5.96) has the form

$$
-D_{i}\left(a^{i}(x, u, D u)\right)=f^{0}
$$

where the divergence is with respect to the metric

$$
\sigma_{i j}(u, x) \text {. }
$$

Let $x^{0}$ be a fixed constant, then we can express the divergence in (9.3) with respect to metric

$$
\sigma_{i j}\left(x^{0}, x\right)
$$

without changing the structure or the properties of the coefficients and $f^{0}$. The volume element in the integrations below will also be defined by this metric.

We now argue similarly as in [5, Section 1] and modify the coefficients of the operator - only a slight adaptation to the present situation is necessary. First, let $\vartheta$ be a smooth real function such that

$$
\vartheta(t)=\left\{\begin{aligned}
t, & m_{1} \leq t \leq m_{2}, \\
-\left(m_{1}+\epsilon_{1}\right), & t \leq-\left(m+\epsilon_{1}\right), \\
\left(m_{2}+\epsilon_{1}\right), & t \geq\left(m_{2}+\epsilon_{1}\right),
\end{aligned}\right.
$$

where $\epsilon_{1}>0$ is chosen small enough to guarantee that

$$
\vartheta(\mathbb{R}) \subset I \text {. }
$$

Secondly, define the metric $\tilde{\sigma}_{i j}(t, x)$ by

$$
\tilde{\sigma}_{i j}(t, x)=\sigma_{i j}(\vartheta(t), x) .
$$

Thirdly, if a vector field $p=\left(p_{i}\right)$ is the gradient of a function $w$,

$$
p=D w,
$$

then set

$$
|p|^{2}=\tilde{\sigma}^{i j} p_{i} p_{j}=\tilde{\sigma}^{i j}(w, x) D_{i} w D_{j} w
$$


Let $\omega, g$ be smooth real functions such that

$$
\omega(t)= \begin{cases}1, & 0 \leq t \leq 1-\frac{\delta^{2}}{2} \\ 0, & t \geq 1-\frac{\delta^{2}}{3}\end{cases}
$$

and assume $g$ to be convex satisfying

$$
g(t)=\left\{\begin{aligned}
0, & 0 \leq t \leq 1-\delta^{2} \\
c t, & t \geq 1-\frac{\delta^{2}}{2}
\end{aligned}\right.
$$

where $c$ is some positive constant. Then, we define

$$
\tilde{a}^{i}(x, t, p)=a^{i}(x, \vartheta(t), p) \omega\left(|p|^{2}\right)+k g^{\prime}\left(|p|^{2}\right) p^{i} .
$$

Here, $k$ is a positive constant. It can be easily verified that

$$
\tilde{a}^{i j}=\frac{\partial \tilde{a}^{i}}{\partial p_{j}}
$$

is uniformly elliptic if $k$ is large enough.

Then, we look at the Dirichlet problem

$$
\begin{aligned}
\tilde{A} w=-D_{i}\left(\tilde{a}^{i}(D w)\right)+\gamma(w-u) & =f^{0}, \\
w_{\left.\right|_{\partial B}} & =0,
\end{aligned}
$$

where $\gamma>0$ is so large that the operator on the left-hand side of (9.15) is uniformly monotone, i.e.,

$$
\left\langle\tilde{A} w_{2}-\tilde{A} w_{1}, w_{2}-w_{1}\right\rangle \geq c_{0}\left\|w_{1}-w_{2}\right\|^{2} \quad \forall w_{1}, w_{2} \in H_{0}^{1,2}\left(B_{R}\right),
$$

where $c_{0}$ is positive. The pairing is the bilinear form between $H_{0}^{1,2}\left(B_{R}\right)$ and its dual space $H^{-1,2}\left(B_{R}\right)$.

Evidently, the solutions of the Dirichlet problem are uniquely determined and since

$$
\tilde{A} u=-D_{i}\left(a^{i}(x, u, D u)\right)=f^{0},
$$

and

$$
\tilde{\sigma}_{i j}(u, x)=\sigma_{i j}(u, x),
$$

we deduce that a solution of (9.15) has to coincide with $u$. It is well known, due to the Calderon-Zygmund inequalities and the De Giorgi-Nash theorem, that (9.15) has a solution

$$
w \in H^{2, p}\left(B_{R}\right)
$$

for any $1<p<\infty$. Indeed, since the operator $\tilde{A}$ is uniformly elliptic and monotone, the unique solution $w \in H_{0}^{1,2}\left(B_{R}\right)$ is then also of class $H^{2,2}\left(B_{R}\right)$, because of the $L^{2}$-estimates, and, since $w=u, w$ is Lipschitz. Furthermore, $D w$ is Hölder continuous with exponent $\beta$ for some $0<\beta<1$ with uniform Hölder norm in

$$
B_{\rho_{0}}\left(x_{0}\right) \subset \bar{B}_{2 \rho_{0}}\left(x_{0}\right) \subset B_{R}
$$


and also in

$$
\Omega\left(x_{0}, \rho_{0}\right), \quad x_{0} \in \partial B_{R},
$$

in view of the De Giorgi-Nash theorem. Hence, the coefficients are continuous and the Calderon-Zygmund inequalities finally yield (9.19). If the data are better we can then apply the Schauder estimates.

Remark 9.1. The norm of $u$ in $H^{2, p}\left(B_{R}\right)$ depends on $R$, but local $H^{2, p}$ norms in

$$
B_{\rho_{0}}\left(x_{0}\right) \subset \bar{B}_{2 \rho_{0}}\left(x_{0}\right) \subset B_{R}
$$

and also in

$$
\Omega\left(x_{0}, \rho_{0}\right), \quad x_{0} \in \partial B_{R},
$$

only depend on $\rho_{0}$ and $p$ but not on $R$, hence, for any $0<\alpha<1$

$$
|u|_{1, \alpha, B_{R}} \leq c
$$

uniformly in $R$.

\section{REFERENCES}

[1] Robert Bartnik, Existence of maximal surfaces in asymptotically flat spacetimes, Comm. Math. Phys. 94 (1984), no. 2, 155-175, doi:10.1007/BF01209300.

[2] John K. Beem, Paul E. Ehrlich, and Kevin L. Easley, Global Lorentzian geometry, second ed., Monographs and Textbooks in Pure and Applied Mathematics, vol. 202, Marcel Dekker Inc., New York, 1996.

[3] Antonio N. Bernal and Miguel Sanchez, Smoothness of time functions and the metric splitting of globally hyperbolic spacetimes, Comm. Math. Physics 257 (2005), 43-50, arXiv:gr-qc/0401112, doi:10.1007/s00220-005-1346-1.

[4] Claus Gerhardt, H-surfaces in Lorentzian manifolds, Commun. Math. Phys. 89 (1983), 523-553, doi:10.1007/BF01214742.

[5] Global $C^{1,1}$-regularity for solutions to quasi-linear variational inequalities, Arch. Rat. Mech. Analysis 89 (1985), 83-92, doi:10.1007/BF00281746.

[6] - Analysis II, International Series in Analysis, International Press, Somerville, MA, 2006.

[7] _ Curvature Problems, Series in Geometry and Topology, vol. 39, International Press, Somerville, MA, 2006.

[8] - Applications of canonical quantum gravity to cosmology, Symmetrie 11 (2019), no. 8, 1005, arXiv:1908.02145, doi:10.3390/sym11081005.

[9] David Gilbarg and Neil S. Trudinger, Elliptic partial differential equations of second order, Grundlehren der Mathematischen Wissenschaften [Fundamental Principles of Mathematical Sciences], vol. 224, Springer-Verlag, Berlin, 1983.

[10] S. W. Hawking and G. F. R. Ellis, The large scale structure of space-time, Cambridge University Press, London, 1973.

[11] N. G. Lloyd, Degree theory, Cambridge University Press, Cambridge-New YorkMelbourne, 1978, Cambridge Tracts in Mathematics, No. 73.

[12] Barrett O'Neill, Semi-Riemannian geometry with applications to relativity., Pure and Applied Mathematics, 103. New York-London: Academic Press. XIII, 1983.

[13] Guido Stampacchia, Équations elliptiques du second ordre à coefficients discontinus, Séminaire de Mathématiques Supérieures, No. 16 (Été, 1965), Les Presses de l'Université de Montréal, Montreal, Que., 1966. 
Ruprecht-Karls-Universität, Institut für Angewandte Mathematik, Im NeuenHeimer Feld 205, 69120 Heidelberg, Germany

E-mail address: gerhardt@math.uni-heidelberg.de

URL: http://www.math.uni-heidelberg.de/studinfo/gerhardt/ 